\title{
Magnetic responsive of paclitaxel delivery system based on SPION and palmitoyl
}

\section{chitosan}

Mona Mansouri ${ }^{1}$, Masoumeh Haghbin Nazarpak $^{2} *$, Atefeh Solouk ${ }^{1}$, Somaye Akbari ${ }^{3}$, Mohammad Mahdi Hasani-Sadrabadi ${ }^{4}$

1 Department of Biomedical Engineering, Amirkabir University of Technology, P.O. Box: 15875/4413, Tehran-159163/4311, Iran.

2 New Technologies Research Center (NTRC), Amirkabir University of Technology, Tehran, 15875-4413, Iran.

3 Department of Textile Engineering, Amirkabir University of Technology, 1P.O. Box: 15875/4413, Tehran-15916/34311, Iran.

4 Parker H. Petit Institute for Bioengineering and Bioscience, G. W. Woodruff School of Mechanical Engineering, Georgia Institute of Technology, Atlanta 30332-0295, GA, United States.

\begin{abstract}
Concerns over cancer treatment have largely focused on chemotherapy and its consequent side effects. Utilizing nanocarriers is thought to be a panacea for mitigating the limitations of chemotherapy, and increasing its safety and efficacy. Magnetically driven Paclitaxel delivery systems are among the commonly investigated types of nanocarriers over the last two decades. In this context, we tried to highlight the application of an AC magnetic field and validate its consequential effects on drug delivery pattern and cell death in such nanodevices. So the aim of this study is to develop an appropriate matrix (Palmitoyl chitosan) co-encapsulated with superparamagnetic iron oxide nanoparticles (SPIONs) and anticancer drug, Paclitaxel (PTX) via the nanoprecipitation process. Synthesized nanoparticles were characterized by Dynamic Light Scattering (DLS) and their magnetic properties were investigated by Vibrating Sample Magnetometer (VSM). At initial loading
\end{abstract}

\footnotetext{
* Corresponding author. Tel.: +982164545194, E-mail address: haghbin@aut.ac.ir
} 
of $10 \mathrm{wt} \%$ Paclitaxel, the maximum loading efficiency of nanoparticles with and without SPIONs was in the range of 69 and $72.3 \%$, respectively. In addition, in vitro release data revealed that by the application of a magnetic field, release kinetic changed to the magnetic responsive pattern. Encapsulating anticancer drug in a synthesized nanosystem not only increased the amount of drug in cancer cells but also enhanced cell death (MCF7) due to hyperthermic effects of SPIONs in the presence of an external magnetic field. In summary, these findings indicate that the resultant nanoparticles may serve as a biocompatible and biodegradable carrier for the precise delivery of powerful cytotoxic anticancer agents such as PTX.

Keywords: Cancer treatment, paclitaxel delivery system, magnetic responsive carrier, hydrophobically modified chitosan, multiple therapies.

\section{Introduction}

Cancer claimed the lives of 8.2 million people in 2012. Based on latest global cancer statistics, breast cancer is the most important cause of death in women in developed and less-developed countries alike [1]. Paclitaxel (Taxol®) is one of the powerful Taxanebased chemotherapy drugs with particular functions on cancer cells. It has a great impact on various types of carcinomas $[2,3]$, however, low aqueous solubility of Paclitaxel has limited its widespread utilization in cancer treatment. For clinical administration, nonionic surfactants such as Cremophor ${ }^{\circledR}$ EL (CrEL; Polyoxyethylene glycerol triricinoleate 35) were utilized in the formulation of Paclitaxel [4, 5]. Undesirables side effects associated with these kinds of solvents on the human body, have made researchers to develop alternative delivery forms of Paclitaxel $[6,7]$. As a result of this, the access to 
safe formulation has become an issue of broad interest in recent years. At this point, utilizing the physical and biochemical properties of nanoparticles can provide considerable benefits to enhance the efficiency of PTX and to decrease adverse implications on human body $[8,9]$.

Magnetic nanoparticles have considerable roles in the biomedical field. With these nanoparticles and an external magnetic field, it is possible to carry therapeutic agents to the target region and keep them at their desired site of action for a longer period of time $[10,11]$. Furthermore, it has been revealed that the magnetic nanoparticles are effective in hyperthermia, localizing heat on particular organs and lastly killing cancer cells by utilizing alternating magnetic fields. Among the magnetic nanoparticles, superparamagnetic iron oxide nanoparticles (SPIONs), due to their ease of preparation, non-toxicity and their ability to tailor their properties for precise applications, especially in tumor treatment, have received wide recognition in recent decades $[12,13]$. Because of hydrophobic-hydrophobic interactions and high tendency to form clusters, the use of naked magnetic nanoparticles in biological environments seems to be ineffective. Larger nanoparticles (> $50 \mathrm{~nm}$ ) appeared to be able to increase the likelihood of non-specific adsorption of opsonins, their uptake by phagocyte system (macrophages) and rapid clearance from the bloodstream [14]. Therefore, the surface coating of these NPs can significantly decrease their limitations and improve their half-life in the blood $[15,16]$. Surface modification of SPIONs can be achieved by different hydrophilic coatings. In the latest studies, there has been increasing interest in using chitosan as a coating material for SPIONs. The popularity of this natural polysaccharide is because of its ability to degrade in biological environments and its affordability $[17,18]$. This biopolymer has active amine and hydroxyl groups, which enable chemical modifications and introduce desired 
functional groups like hydrophobic moieties to the main backbone such as the conjunction of glycerol monooleate [19], deoxycholic acid [20] and 5 $\beta$-cholanic acid [21]. In addition, $\mathrm{N}$-acylation of chitosan with various long-chain fatty acids, such as oleic acid [22], linoleic acid [23], and palmitic acid [24, 25], have been used as a hydrophobic moiety for the formation of a hydrophobic matrix and encapsulation of lipophilic drugs such as Paclitaxel [26]. Recently, Majedi et al. [27] revealed that nanoparticles composed of Palmitic acid N-hydroxysuccinimide ester can be easily self-assembled at physiological $\mathrm{pH}$ and are helpful in sustaining the PTX release from its hydrophobic matrix. This method is of great importance from toxicity point of view due to the fact that selfaggregation of polymeric chains in an aqueous media eliminates the need for chemical crosslinkers. The objective of this study was to analyze the delivery and therapeutic efficacy of PTX by encapsulating it into composite nanocarriers and then delivering them to target cells through an external magnetic field. In this study, the effects of an external AC magnetic field on drug release profile as well as cancerous cell death were reported.

\section{Materials and Methods}

\subsection{Materials}

Ferrous chloride tetrahydrate $\left(\mathrm{FeCl}_{2} \cdot 4 \mathrm{H}_{2} \mathrm{O}\right)$ and ferrous chloride hexahydrate $\left(\mathrm{FeCl}_{3} \cdot 6 \mathrm{H}_{2} \mathrm{O}\right)$ were purchased from Merck. Chitosan (MW= $50-190 \mathrm{kDa}$, degree of deacetylation of 75-85\%) was obtained from Sigma Aldrich. Palmitic acid Nhydroxysuccinimide ester and anti-cancer drug Paclitaxel (PTX) were purchased from Sigma-Aldrich. Glacial acetic acid, sodium hydroxide, ammonium hydroxide and ethanol were obtained from Merck. All other chemicals were of analytical grade. 


\section{2 . Synthesis of SPIONs}

SPIONs were prepared as reported by Petri-Fink et al. [28]. Briefly $0.086 \mathrm{M} \mathrm{FeCl}_{3} \cdot 6 \mathrm{H}_{2} \mathrm{O}$ and $0.043 \mathrm{M} \mathrm{FeCl}_{2} \cdot 4 \mathrm{H}_{2} \mathrm{O}$ were prepared in double distilled water. Thereafter, the mixture of iron solutions was sonicated (Elma - E30H, Germany) for $30 \mathrm{~min}$. This solution was added drop-wise to the stirring ammonia solution and mixed at $25^{\circ} \mathrm{C}$. The dark-colored precipitation was purified using magnetic separation and washed several times with deionized water until the $\mathrm{pH}$ reached the value of 7 . The resultant material was refluxed in a mixture of $0.8 \mathrm{M}$ nitric acid and $0.21 \mathrm{M}$ aqueous $\mathrm{Fe}\left(\mathrm{NO}_{3}\right)_{3} .9 \mathrm{H}_{2} \mathrm{O}$ for $1 \mathrm{~h}$, from which, nitric oxide was formed. At the next stage, the system was cooled down and the remaining liquid was removed and $100 \mathrm{ml}$ of ultra-pure water was added to the mixture to redisperse the precipitate. Finally, the brown suspension was dialyzed for 2 days against $0.01 \mathrm{M}$ nitric acid, and stored at $4^{\circ} \mathrm{C}$.

\subsection{Preparation of palmitoyl chitosan}

Hydrophobically-modified chitosan was synthesized by a single-step reaction [24]. For this purpose, $1 \mathrm{~g}$ of chitosan was dissolved in $1 \%(\mathrm{w} / \mathrm{v})$ aqueous acetic acid solution (50 $\mathrm{mL}$ ) and then diluted by the addition of deionized water. The solution was filtered with a $0.45 \mu \mathrm{m}$ nylon membrane filter. Then, the $\mathrm{pH}$ was adjusted to 6 by the slow addition of 1 $\mathrm{N} \mathrm{NaOH}$. A solution of 100-400 mg palmitic acid N-hydroxysuccinimide ester in absolute ethanol was added drop-wise to the chitosan solution at $98{ }^{\circ} \mathrm{C}$ under reflux and reacted for

$36 \mathrm{~h}$. Subsequently, the solution was cooled down to room temperature and precipitated by adjusting the solution $\mathrm{pH}$ to 9.0. The precipitated polymer was then filtered and vacuumdried at room temperature. 
For bulk synthesis of HMCS nanoparticles, $2.5 \mathrm{mg} / \mathrm{mL}$ HMCS was dispersed in $1 \% \mathrm{w} / \mathrm{v}$ acetic acid solution and stirred. Drop-wise addition of this solution to water while stirring resulted in the formation of nanoparticle. Actually, after increasing the $\mathrm{pH}$ level, HMCS molecules were precipitated in the solution.

\subsection{Preparation of SPION coated palmitoyl chitosan}

Palmitoyl chitosan (100mg) was dissolved in acetic acid solution and sonicated for 15 min. Then SPION solution in ethanol was added to the previous solution, and the suspension was vigorously stirred for 30 min to ensure the even coating of the chitosan onto the nanoparticles. A volume of $1 \mathrm{~N} \mathrm{NaOH}$ was slowly added to the suspension to precipitate the coated nanoparticles. The resulting SPION-chitosan nanoparticles were recovered from the suspension with a strong magnet. Finally, they were washed with deionized water several times.

For drug-loaded nanoparticles, Paclitaxel (10 wt\%) was dissolved in acidic water (pH 4.5) and mixed with the polymeric solution (with-/out SPIONs). Then, the mixture was added dropwise into the water $(\mathrm{pH}=7.4)$ under gentle stirring, allowing the formation and precipitation of nanoparticles. Detailed chemical compositions of nanoparticles are listed in Fig. 1.

\subsection{Drug loading and in vitro drug release}

In vitro release studies were performed by dispersion of $1 \mathrm{mg}$ PTX-loaded nanoparticles in $1 \mathrm{~mL}$ of phosphate buffered saline (1X PBS, $\mathrm{pH} 7.4$ ). Then the solutions were placed into dialysis cartridges (3500 Da, Thermo Scientific, Rockford, IL) and suspended in 1 L PBS 
buffer. Samples were gently shaken at $37{ }^{\circ} \mathrm{C}$ in a water bath at $100 \mathrm{rpm}$. At predetermined time intervals, 3 aliquots $(1 \mathrm{~mL})$ were taken from the release medium, and their PTX concentrations were determined by HPLC. After each sampling, an equivalent volume of fresh PBS was replenished immediately in order to maintain the sink condition. Similarly, sampling in the presence of an applied magnetic field was performed according to the above-mentioned procedure. The strength of the applied AC magnetic field was 0.2 Tesla.

The measured drug concentration can be corrected for the drug removed in the previous samples by applying the following equation [29]:

$$
C_{n}^{\prime}=C_{n}\left(\frac{V_{T}}{V_{T}-V_{S}}\right)\left(\frac{C_{n-1}^{\prime}}{C_{n-1}}\right)
$$

Where, $C_{n}$ ' is the corrected concentration of the nth sample, $C_{n}$ is the measured concentration of PTX in the nth sample, $C_{n-1}$ is the measured concentration of the (n-1)th sample, $V_{T}$ is the volume of the receiver fluid, and $V_{S}$ represents the volume of the drawn sample $(1 \mathrm{~mL})$.

The removed samples were stored at $-20{ }^{\circ} \mathrm{C}$ until they were analyzed by HPLC. Chromatographic separation was achieved using a reverse phase $\mathrm{C} 18$ column as a stationary phase. The mobile phase consisted of acetonitrile/water $(60: 40 \mathrm{v} / \mathrm{v})$. The flow rate was set to $1.0 \mathrm{~mL} / \mathrm{min}$ and UV detection wavelength was $230 \mathrm{~nm}$. The concentration of PTX was determined based on a calibration curve. Based on this, a standard linear-fit curve of free PTX was created by plotting the UV-Vis absorbance at $230 \mathrm{~nm}$ of several PTX solutions of known concentrations. Then the lyophilized PTX-loaded nanoparticles 
$(1 \mathrm{mg})$ were dissolved in THF and the PTX concentrations were measured using the same HPLC protocol as earlier mentioned. The drug loading content and loading efficiency were determined by applying the following equations:

PTX loading content $=($ weight of the loaded PTX/weight of the NPs $) \times 100($ Eq. 2$)$

PTX loading efficiency $=($ amount of PTX in the NPs /initial amount of the PTX $) \times 100$ (Eq.3)

\subsection{In vitro cytotoxicity assay}

MCF-7 cells were grown in DMEM medium supplemented with $10 \%$ fetal bovine serum and antibiotics. The cells were seeded into 96 -well plates at $10 \times 10^{3}$ cells/well and incubated for $24 \mathrm{~h}$ in humidified atmosphere of $5 \% \mathrm{CO}_{2}$ and at $37{ }^{\circ} \mathrm{C}$. Then cells were exposed to free Paclitaxel and Paclitaxel- loaded nanoparticles. For Paclitaxel treatment, a stock solution of Paclitaxel $(10 \mathrm{mg} / \mathrm{mL}$ in DMSO) was diluted to define concentration in the culture medium. To determine if the prepared carriers exhibit cytotoxic effects, nanoparticles (with/-out SPION) were suspended in DMEM, supplemented with FBS and antibiotics at different concentrations and then applied to culture wells. A negative test control containing only growth medium was utilized. After treating the samples for $72 \mathrm{~h}$, the MTT solution (5 mg/mL in phosphate buffer $\mathrm{pH}$ 7.4) was added, and the cells were incubated for $2 \mathrm{~h}$. The absorbance of reduced MTT (formazan) solubilized in DMSO was then measured using a plate reader (Safire II, Tecan Sales Switzerland AG, Mannedorf, $\mathrm{CH})$ at $570 \mathrm{~nm}$. The relative cell viability (\%) was calculated by the following equation:

$$
\frac{\left[A_{\text {sample }}\right]}{\left[A_{\text {control }}\right]} \times 100
$$


Where $\left[\mathrm{A}_{\text {sample }}\right]$ is the absorbance of the test sample and $\left[\mathrm{A}_{\text {control }}\right]$ is the absorbance of the control sample. The results are presented as a mean \pm standard deviation $(\mathrm{SD})(\mathrm{n}=3)$ and were statistically analyzed using unpaired t-tests. Differences with P-values $<0.05$ were considered statistically significant.

\subsection{Characterization of nanoparticles}

The crystalline phase of SPION was confirmed using X-ray Diffraction (XRD, Philips PW-3710, Netherlands) with radiation of $\mathrm{Cu}(\lambda=0.15406 \mathrm{~nm})$. In this regard, a continuous scan mode was used to collect $2 \theta$ data from 10 to $90^{\circ}$. Magnetic properties of prepared samples before and after coating were measured using Vibrating Sample Magnetometer (VSM, ADE 4HF, US) at room temperature. The prepared hydrophobically modified chitosan (HMCS) was analyzed by Fourier Transform Infrared (FTIR) spectroscopy, and the Degree of Substitution (DS) was determined by the ninhydrin assay. Briefly, $0.3 \mathrm{mg}$ HMCS was dissolved in an aqueous acetic acid and thoroughly stirred. Subsequently, 0.5 $\mathrm{mL}$ of acetic acid/acetate buffer (4 M, pH 5.5) was added into different concentrations of HMCs solution $(0.1-0.5 \mathrm{~mL})$. Then, ninhydrin regent $(1 \mathrm{~mL})$ was added, and test tubes were placed in a boiling-water bath for $20 \mathrm{~min}$. Thereafter, the solutions were cooled and their absorbance at $570 \mathrm{~nm}$ was read (UV-Vis Spectrophotometer, Analytikjena, SPECORD 210, Germany). The acetic acid/acetate buffer was utilized as a blank and the unmodified chitosan solution was used as a control. Zeta potential and hydrodynamic particle diameter (with/-out SPION) were determined by dynamic light scattering measurements in water at $25^{\circ} \mathrm{C}(\mathrm{pH}=7.4)$ using a Malvern Zetasizer (Zetasizer 3000HS, Malvern Instruments Ltd, Worcestershire, UK). The amount of magnetite nanoparticles in resultant nanocomposites was estimated using ICP-OES (Inductively Coupled Plasma - 
Optical emission spectroscopy) after digestion with $\mathrm{HCl}$. Finally, the morphology of the naked and polymer coated magnetite nanoparticles were investigated by Scanning Electron Microscopy (SEM).

\section{Results and Discussion}

Characterization and crystal structure of naked SPIONs were successfully analyzed by XRD diffractogram (Fig.2). Characteristic peaks at (1, 1 1), (2 2 0), (3 1 1 1), (4 0 0 0), (4 2 2), ( $\left.\begin{array}{lll}5 & 1 & 1\end{array}\right),\left(\begin{array}{lll}4 & 4 & 0\end{array}\right),\left(\begin{array}{lll}5 & 3 & 3\end{array}\right)$, which are in agreement with the standard pattern for $\mathrm{Fe}_{3} \mathrm{O}_{4}$ with a cubic inverse spinel structure (JCPDS file No. 19-0629) were observed [30].

Hydrophobically modified chitosan was synthesized via the reaction of chitosan with Palmitic acid N-hydroxysuccinimide ester (Fig. 3(a)). As seen from Fig. 3(b), the native chitosan shows the broad peak in the region of $3400 \mathrm{~cm}^{-1}$ for hydroxyl group. Stretching C-H bond (SP3) was exhibited at $2875 \mathrm{~cm}^{-1}$ along with the primary amino groups bending vibration at $1651 \mathrm{~cm}^{-1}$. After grafting hydrophobic palmitoyl onto the amine groups of chitosan, new signals at 3003.45 and $2936.91 \mathrm{~cm}^{-1}$ appeared which were assigned to $-\mathrm{CH}_{2}$ asymmetric and symmetric stretch, respectively. Asymmetric stretching vibrations generate a larger dipole moment; hence they occur at higher frequencies than the symmetric one. Prominent peaks at 1588.03 and $1427.67 \mathrm{~cm}^{-1}$ were attributed to $\mathrm{N}-\mathrm{H}$ bending vibrations and $\mathrm{C}=\mathrm{O}$ stretching, respectively; which corresponded to the amide bond. These results indicated that the palmitoyl chitosan was obtained, and the reaction is highly selective toward $\mathrm{N}$-acylation. 
The amount of hydrophobic content in amphiphilic polymer was measured by the color intensity produced by ninhydrin assay and using standard curve. Values of 4.4, 9.7, 15.1 and 22.5 were the determined degree of substitution (DS) on chitosan.

Long hydrophobic chains of chitosan, after being exposed to aqueous media, spontaneously formed aggregates to produce thermodynamically stable micelles with hydrophobic cores, whereas hydrophilic chitosan chains greatly contribute to form an outer shell, leading to the fabrication of polymeric micelles [31]. Palmitoyl groups play a significant role as a physical cross linker (i.e. non-covalently) between polymeric chains [32]. As a matter of fact, the nature of the material such as intra- and/or inter-molecular hydrophobic interaction and polymer chain lengths have a conspicuous impact on the formation of nanoparticles in the absences of toxic and chemical crosslinkers [33].

Blood vessels associated with tumors possess deficient pericytes, excessive endothelial cell proliferation and discontinuous basement membrane with irregular gaps, ranging from 380 to $780 \mathrm{~nm}$. So it is important to establish suitable engineered nanoparticles for particular purposes, especially in cancer therapy. Reducing the size of the nanoparticles to desired value facilitates nanoparticles' endocytosis and cellular uptake mechanism thereby enhancing the tumor penetration and accumulation. Likewise, nanoparticles with large size (>300 $\mathrm{nm}$ ) tend to be phagocytize by macrophage while very small particles $(<10 \mathrm{~nm})$ are more likely to undergo renal elimination. Therefore, the size of nanoparticles also determines their fate in the human body [34-37]. Providing access to the specific site that is not available to larger particles is another overt advantage of utilizing small size. In a simple term, evaluating particle size is of great significance in designing novel nanodevices for diagnostic and therapeutic applications [38, 39]. There are diverse factors that have marked effect on particle's size. It is proposed that the molecular weight of 
chitosan has an outstanding impact on ultimate dimensions of nanoparticles. The lower molecular weight is equal to the shorter polymeric chain and smaller particle size [40, 41]. In addition, it has been proved that physical characteristics of the nanoparticles, such as size and stability, depend on the degree of fatty acid substitution [42]. With increase in the degree of substitution, due to stronger hydrophobic interactions, the tendency to aggregate increases and this phenomenon leads to obvious downward trend in particle size [24] which is clearly shown in Fig.5(a).

During the self-assembly process, as hydrophobic chains form the core region, hydrophobic SPIONs and poorly water-soluble PTX can be encapsulated by the hydrophobic domains of micelles (Fig.4). The size of nanoparticles was found to change slightly when SPIONs were entrapped inside the polymeric chains (p>0.05) (Fig.5(a)). While their zeta potential at $\mathrm{pH} 7.4$, fluctuated around 4 and had a steady change. Positive zeta potential is as a result of the presence of the charged amino group of chitosan on the surface of SPIONs (Fig.5 (b)). The low zeta values indicated that nanoparticles possessed low electric charges on their surfaces, which affect their prolong circulation as a result of the weak repellent forces between them [43]. However, an acceptable stability was observed during the experiment.

It is apparent from Fig.5(c) that the mean diameters of the nanoparticles increased slightly after drug loading. Although the dimension of nanoparticles was expected to show a dramatic increase after drug loading [44-46], their size is pale in comparison to their size before encapsulating PTX ( $>00.05)$. Moreover, in some samples, gradual reduction in the size of drug-loaded nanoparticles was observed. The hydrophobic nature of drug acts noticeably in this regard. After encapsulating PTX, the intermolecular hydrophobic interactions in the nanoparticle's core increased, followed by a slight change in size. 
Therefore, their characteristics remain desirable for medical applications, even after drug loading.

VSM measurements demonstrated the super paramagnetic behavior of SPIONs before and after being coated by chitosan. This study is important in estimating the change in magnetic properties occurring after coating. As shown in Fig.5 (d), there are no significant remanence and coercivity in the hysteresis loop of samples, indicating that both nanoparticles were super-paramagnetic. When all magnetic domains align themselves with the direction of the applied field, saturation magnetisation is obtained. On the other hand, saturation remanence occurs when the magnetisation does not completely revert back to zero. The force used to bring magnetisation down again to the former situation is called coercivity [47].

The magnetic saturations for naked SPIONs were approximately $74 \mathrm{emu} / \mathrm{g}$ while for coated SPION, the magnetic saturation reached just over $40 \mathrm{emu} / \mathrm{g}$. Higher saturation magnetisation stems from pure cubic $\mathrm{Fe}_{3} \mathrm{O}_{4}$ spinel phase, the absence of non-magnetic impurities and small size. On the contrary, the lower saturation magnetisation is attributed to the polymeric surface layer. These results are consistent with previous studies which proved that the saturation magnetisation of SPIONs was markedly reduced after being encapsulated by polymers [47-49]. One sure way to ensure SPIONs' colloidal stability in biological media and prolong their circulation time in blood is surface coating with hydrophilic polymers [50].

Fig.6 illustrates a comparison between bare and coated SPIONs. As expected, naked SPIONs due to small size and high surface energy, exhibited a strong tendency to aggregation, while fewer cluster was observed after coating them with chitosan. 
In the history of PTX, the focus has always been on solving its solubility limits in aqueous media. The lack of ionizable functional groups and enormous quantity of hydrophobic building blocks are some challenges in the formulation of PTX, which have limited its widespread applications in the biomedical field [51-53]. Low PTX loading and poor stability of some nanocarriers intrigue many researchers to work on these problems and introduce qualified nanosystems. In this study, PTX was effectively encapsulated into nanocarriers with promising loading efficiency in the range of 69 and $72.3 \%$ for nanoparticles with and without SPIONs, respectively (Fig.7 (a)). Moreover, an AC magnetic field was utilized to trigger the release of PTX. The results of in vitro PTX release profiles in an aqueous environment in the presence and absence of magnetic field are shown in Fig.7. It should be noted that the fourth sample with $22.5 \%$ degree of substitution was not utilized for overall assessment of toxicity and release profile as a result of rapid agglomeration and poor stability in aqueous media.

As seen in Fig.7 (b), in the absence of magnetic field, release profile rose sharply during $48 \mathrm{~h}$. From the third day onwards, the profile remained reasonably steady. These results agree with previous studies reporting a biphasic drug release profile of PTX $[54,55]$. The rapid release in the first phase can be attributed to the diffusion of PTX molecules close to the surface, from a region of high-concentration to a region of low-concentration, following Fick's laws [56, 57]. Actually, these molecules could not be entrapped in the deeper part of the particles during preparation, so they discharge promptly into PBS. After that, it takes more time for drug molecules to travel from the inner part to the surface and leave the nanoparticle. Among synthesized carriers, nanoparticles with DS=15.1 showed a nearly slower release profile next to other samples. This phenomenon could be a result of 
the compact structure of polymer chains and the lack of conformational freedom between them, leading to decline in the apparent diffusion rate [58, 59].

Among the second group, the third sample (DS=15.1) was chosen for the evaluation of release profile and in vitro cytotoxic effect. This category clearly has high magnetic contents, which allow it to extremely respond to an external magnetic stimulation (Fig.5 (c)). As shown in Fig.7 (c), in the absence of a magnetic field, the release rate steadily increased for 4 days, then it slowed down and remained stable until the end of the examination. By applying an external magnetic field $(0.2 \mathrm{~T})$, the release profile increased dramatically, reaching a peak in $24 \mathrm{~h}$; in a way that it was completed in 5.5 days. The findings showed that in the absence of a magnetic field, the drug incorporated into the nanoparticles was mainly released by diffusion, while for the other group; magnetic components enabled the tailorable release kinetics through the use of a magnetic field. Drug release rate can be substantially improved by being exposed to a stimulus. Temperature, $\mathrm{pH}$ and magnetic fields all serve these purposes and can promote the drug release from nanoparticles. In previous studies, alternative magnetic fields with different frequencies were used to investigate the drug release from various systems [60-62]. Magnetic-sensitive drug delivery systems will retain the drug at physiological conditions and release their payload as a consequence of external stimuli. In this study, since PTX was not chemically bound to the functional groups, the heat generated by magnetic particles was utilized to create cavities within a polymeric matrix which led to an acceleration in diffusion of encapsulated drug molecules from openings into aqueous media $[63,64]$.

The kinetics of PTX release from nanoparticles were determined by the following equation proposed by Higuchi based on Fick's second law $[65,66]$ : 


$$
\frac{M_{t}}{M_{\infty}}=6\left(\frac{D t}{\pi R_{h}{ }^{2}}\right)^{1 / 2}
$$

In this equation, $M t / M \infty$ is the fraction of released PTX at time $t, D$ is the diffusion coefficient of the drug molecules and $R$ is the hydrodynamic radius of synthesized nanoparticles. From this model, D was calculated.

The results of the diffusion coefficient of the drug molecules from nanoparticles are presented in Fig.7 (d). In the polymeric nanoparticles (without SPION), diffusion coefficient rapidly declined with increase in the amount of hydrophobic segments in the nanoparticles. This issue is clearly linked to the particle's size. The smaller the size of the nanoparticles, the lower the diffusion will become. So the smallest particles $(\mathrm{DS}=15.1)$ tend to have lower diffusion coefficient in comparison with their counterparts because they possess less free space for transporting drug molecules. Similarly, this trend occurs in the rest of samples. The larger nanoparticles also results in the subsequent increase in the $\mathrm{D}$ value. That might be ascribed to the degree of swelling and the volume of the pores available for drug diffusion due to the protonation of more amine groups [67]. All evidence proved that after applying an external magnetic field, the transport mechanism does not follow Fick's law and changes to the magnetic responsive mechanism. This result is consistent with the release profile and confirms them. Therefore, tracing the D value can be seen as an overall indication of release rate determining how long it takes for the drug to discharge into a physiological environment.

Moreover, cell viability MTT assay was carried out on breast cancer cell lines (MCF-7) for studying the tumor response to PTX- loaded nanoparticles compared with free PTX, 
and to find out if the prepared nanoparticles exhibit any cytotoxic effects on MCF-7 cells, in the presence of a magnetic field. After $72 \mathrm{~h}$ incubation of drug- free nanoparticles with cancer cells, no sign of toxicity was observed in this group at low and high concentrations of samples alike.

Presented graph (Fig.8), makes a comparison between PTX-loaded nanoparticles and free PTX effect on cancer cells, before and after applying an external magnetic field. As shown in the figure, the cell death via synthesized nanoparticles is considerably higher than free PTX ( $\mathrm{p}<0.05$ ). Nanoparticles (with/ out SPIONs) increased endocytosis process of PTX into cells as a result of their small size and positive surface charge thereby improving the concentration of drug in the cells, which markedly increases the cytotoxic effects of the anticancer drug. Undoubtedly, the effect of chitosan should not be underestimated for this purpose. Chitosan is among those polymers that is believed to potentially disrupt intercellular tight junctions and can lengthen the residence time of delivery systems at specific sites [33].

Furthermore, it seems that heat derived from nanocomposites under an alternative magnetic field induces cell death in the local area. Rapid changes in orientation of dipole moments are responsible for the generating of heat from nanocarriers, which has a detrimental impact on cancer cells. As a matter of fact, denaturation of membrane and cytoplasmic proteins together with the alteration of DNA synthesis and repair result in cell killing through hyperthermia. In addition, focusing heat on the tumor area is found to cause a reduction in blood flow, improve tumor oxygenation and enhance vascular permeability making cancer cells more sensitive to therapeutic agents such as chemotherapy drugs $[47,68,69]$. 
Therefore, the combination of SPIONs and chitosan in the same nanocarrier has made it more likely to take full advantages of controlled drug delivery and hyperthermia simultaneously, and also benefit from the synergetic effects of the multiple therapies.

\section{Conclusion}

In summary, in this study, PTX loaded nanocomposites based on palmitoyl chitosan and SPION with significant magnetic properties; appropriate surface charge and stability were successfully developed by the nanoprecipitation method. The effects of various degrees of substitution (DS) of palmitoyl groups on physical properties and encapsulation efficiency of nanoparticles were investigated. Among the prepared nanoparticles, samples with $15.1 \%$ DS demonstrated a significant drug- loading capacity, as well as high encapsulation of iron oxide nanoparticles. Further and even more importantly, the impact of an AC magnetic field on the release profile and cancerous cell death was evaluated. In the absence of a magnetic stimulation, similar release patterns were seen from samples in $\mathrm{pH}$ 7.4. The drug transport was dependent on swelling followed by diffusion; whereas after applying a magnetic field, the drug release rate was highly improved and happened much faster, as a result of partial structural loosening. Furthermore, using an external magnetic field, markedly affected the internalization of synthesized nanoparticles, compared with free PTX. Therefore, drug molecules can accumulate in cells and this happening leads to noticeable cell death.

\section{Acknowledgement}

This study was supported by the Iran National Science Foundation (INSF) Grant No. 93041804. 


\section{References:}

1. T. Lindsey A, et al. Global cancer statistics, 2012. CA: a cancer journal for clinicians, 2015, 65, 87-108.

2. D. Cella, et al. Measuring the side effects of taxane therapy in oncology. Cancer, 2003, 98, 822-831.

3. M. Skwarczynski, Y. Hayashi, and Y. Kiso, Paclitaxel prodrugs: toward smarter delivery of anticancer agents. Journal of medicinal chemistry, 2006, 49, 72537269 .

4. T.Hamaguchi, et al. NK105, a paclitaxel-incorporating micellar nanoparticle formulation, can extend in vivo antitumour activity and reduce the neurotoxicity of paclitaxel. British journal of cancer, 2005, 92, 1240-1246.

5. DA.Yardley, nab-Paclitaxel mechanisms of action and delivery. Journal of Controlled Release, 2013, 170, 365-372.

6. JS. Sohn, JI. Jin, M. Hess and BW. Jo, Polymer prodrug approaches applied to paclitaxel. polymer chem, 2010, 1, 778-792.

7. Y.Lee, et al. Paclitaxel-Loaded Polymer Nanoparticles for the Reversal of Multidrug Resistance in Breast Cancer Cells. Advanced Functional Materials, 2011, 21, 4211-4218.

8. N. Kamaly, et al. Targeted polymeric therapeutic nanoparticles: design, development and clinical translation. Chemical Society Reviews, 2012, 41, 29713010 .

9. CY. Ang, SY . Tan, and Y. Zhao, Recent advances in biocompatible nanocarriers for delivery of chemotherapeutic cargoes towards cancer therapy. Organic \& biomolecular chemistry, 2014, 12, 4776-4806.

10. M. Muthiah, IK. Park, and CS. Cho, Surface modification of iron oxide nanoparticles by biocompatible polymers for tissue imaging and targeting. Biotechnology advances, 2013, 31, 1224-1236.

11. S. Mura, J. Nicolas, and P. Couvreur, Stimuli-responsive nanocarriers for drug delivery. Nature materials, 2013, 12, 991-1003.

12. JA. Barreto, et al. Nanomaterials: applications in cancer imaging and therapy. Advanced Materials, 2011, H18-H40 .

13. M. Hoffman-Amtenbrink, B. Von Rechenberg, and H. Hofmann, Superparamagnetic nanoparticles for biomedical applications. 2009 .

14. SA. Wahajuddin, Superparamagnetic iron oxide nanoparticles: magnetic nanoplatforms as drug carriers. International journal of nanomedicine, 2012, 7, 3445 .

15. EK. Schlachter, et al. Metabolic pathway and distribution of superparamagnetic iron oxide nanoparticles: in vivo study. International journal of nanomedicine, $2011,6,1793$. 
16. W.Wu, Q. He, and C. Jiang, Magnetic iron oxide nanoparticles: synthesis and surface functionalization strategies. ChemInform, 2009, 40,i.

17. L. Hu, Y. Sun, and Y. Wu, Advances in chitosan-based drug delivery vehicles. Nanoscale, 2013, 5, 3103-3111.

18. KO. Doh, and Y. Yeo, Application of polysaccharides for surface modification of nanomedicines. Therapeutic delivery, 2012, 3, 1447-1456.

19. L.Wang, et al. In vitro and in vivo evaluation of chitosan graft glyceryl monooleate as peroral delivery carrier of enoxaparin. International journal of pharmaceutics, 2014, 471, 391-399.

20. Z. Shi, et al . Nanoparticles of deoxycholic acid, polyethylene glycol and folic acid-modified chitosan for targeted delivery of doxorubicin. Journal of Materials Science: Materials in Medicine, 2014, 25, 723-731.

21 . JH. Park, et al. Self-assembled nanoparticles based on glycol chitosan bearing $5 \beta$ cholanic acid for RGD peptide delivery. Journal of Controlled Release, 2004, 95, 579-588.

22. CM. Lee, et al. Oleyl-chitosan nanoparticles based on a dual probe for optical/MR imaging in vivo. Bioconjugate chemistry, 2011, 22, 186-192

23. CG. Liu, et al. Linolenic acid-modified chitosan for formation of self-assembled nanoparticles. Journal of agricultural and food chemistry, 2005, 53, 437-441 .

24. YL. Chiu, et al. The characteristics, cellular uptake and intracellular trafficking of nanoparticles made of hydrophobically-modified chitosan. Journal of Controlled Release, 2010, 146, 152-159.

25. GB. Jiang, et al. Novel polymer micelles prepared from chitosan grafted hydrophobic palmitoyl groups for drug delivery. Molecular pharmaceutics, 2006, $3,152-160$

26. C.Le Tien, et al. N-acylated chitosan: hydrophobic matrices for controlled drug release. Journal of Controlled Release, 2003, 93, 1-13.

27. F.S. Majedi, et al. On-Chip Fabrication of Paclitaxel-Loaded Chitosan Nanoparticles for Cancer Therapeutics. Advanced Functional Materials, 2014, 24, 432-441.

28. A. Petri-Fink,et al. Development of functionalized superparamagnetic iron oxide nanoparticles for interaction with human cancer cells. Biomaterials, 2005, 26, 2685-2694.

29. WL. Hayton, and T. Chen, Correction of perfusate concentration for sample removal. Journal of pharmaceutical sciences, 1982, 71, 820-821

30. J. Qu, et al. Preparation of Fe $3 \mathrm{O}$ 4-chitosan nanoparticles used for hyperthermia. Advanced Powder Technology, 2010, 21, 461-467.

31. JH. Park, et al. Self-assembled nanoparticles based on glycol chitosan bearing hydrophobic moieties as carriers for doxorubicin: in vivo biodistribution and antitumor activity. Biomaterials, 2006, 27, 119-126. 
32. G. Liang, et al. Preparation, characterization and pharmacokinetics of N-palmitoyl chitosan anchored docetaxel liposomes. Journal of pharmacy and pharmacology, 2007, 59, 661-667.

33. SK. Nitta, and K. Numata, Biopolymer-based nanoparticles for drug/gene delivery and tissue engineering. International journal of molecular sciences, 2013, 14, $1629-1654$

34. M.E. Davis and D.M. Shin, Nanoparticle therapeutics: an emerging treatment modality for cancer. Nature reviews Drug discovery, 2008, 7, 771-782 .

35. De Jong, W.H. and P.J. Borm, Drug delivery and nanoparticles: applications and hazards. International journal of nanomedicine, 2008, 3, 133 .

36. C. He et al. Effects of particle size and surface charge on cellular uptake and biodistribution of polymeric nanoparticles. Biomaterials, 2010, 31, 3657-3666.

37. A.S. Thakor and S.S. Gambhir, Nanooncology: the future of cancer diagnosis and therapy. CA: a cancer journal for clinicians, 2013, 63, 395-418.

38. W. Jiang et al. Nanoparticle-mediated cellular response is size-dependent. Nature nanotechnology, 2008, 3, 145-150.

39. E.C. Dreaden et al. Size matters: gold nanoparticles in targeted cancer drug delivery. Therapeutic delivery, 2012, 3, 457-478.

40. M. Huang, E. Khor, and L.Y. Lim, Uptake and cytotoxicity of chitosan molecules and nanoparticles: effects of molecular weight and degree of deacetylation. Pharmaceutical research, 2004, 21, 344-353.

41. Yang and M.H Hon, The effect of the molecular weight of chitosan nanoparticles and its application on drug delivery. Microchemical Journal, 2009, 92, 87-91 .

42. F. Andrade, et al. Chitosan-grafted copolymers and chitosan-ligand conjugates as matrices for pulmonary drug delivery. International Journal of Carbohydrate Chemistry, 2011, 2011.

43. M. Shen, et al. The synthesis and characterization of monodispersed chitosancoated $\mathrm{Fe} 3 \mathrm{O} 4$ nanoparticles via a facile one-step solvothermal process for adsorption of bovine serum albumin. Nanoscale research letters, 2014, 9, 1-8.

44. Y. Hamano, Amino-acid homopolymers occurring in nature. Springer Science \& Business Media, 2010, 15.

45. H.Y. Kim , et al. Paclitaxel-incorporated nanoparticles using block copolymers composed of poly (ethylene glycol)/poly (3-hydroxyoctanoate). Nanoscale research letters, 2014, 9, 1-10.

46. M. Esfandyari-Manesh, et al. Improved anticancer delivery of paclitaxel by albumin surface modification of PLGA nanoparticles. DARU Journal of Pharmaceutical Sciences, 2015, 23, 1 .

47. A. Hervault, and NTK. Thanh, Magnetic nanoparticle-based therapeutic agents for thermo-chemotherapy treatment of cancer. Nanoscale, 2014, 6, 11553-11573. 
48. M. Tajabadi, M.E. Khosroshahi, and S. Bonakdar, An efficient method of SPION synthesis coated with third generation PAMAM dendrimer. Colloids and Surfaces A: Physicochemical and Engineering Aspects, 2013, 431, 18-26.

49. Y. Xiao, et al. high molecular weight chitosan derivative polymeric micelles encapsulating superparamagnetic iron oxide for tumor-targeted magnetic resonance imaging. International journal of nanomedicine, 2015, 10, 1155 .

50. N. Lee, and T. Hyeon, Designed synthesis of uniformly sized iron oxide nanoparticles for efficient magnetic resonance imaging contrast agents. Chemical Society Reviews, 2012, 41, 2575-2589.

51. S. Singh and A.K. Dash, Paclitaxel in cancer treatment: perspectives and prospects of its delivery challenges. Critical Reviews ${ }^{\mathrm{TM}}$ in Therapeutic Drug Carrier Systems, 2009, 26.

52. MS. Surapaneni, SK. Das, and NG. Das, Designing Paclitaxel drug delivery systems aimed at improved patient outcomes: current status and challenges. ISRN pharmacology, 2012, 2012.

53. C. Nehate, et al. Paclitaxel formulations: challenges and novel delivery options. Current drug delivery, 2014, 11, 666-686.

54. U. Westedt, et al. Poly (vinyl alcohol)-graft-poly (lactide-co-glycolide) nanoparticles for local delivery of paclitaxel for restenosis treatment. Journal of controlled release, 2007, 119, 41-51.

55. B. Wang, et al. Paclitaxel and etoposide co-loaded polymeric nanoparticles for the effective combination therapy against human osteosarcoma. Nanobiotechnol, 2015, $13,1-11$.

56. W. Pan and Z. Yang, Thermoreversible Pluronic $®$ F127-based hydrogel containing liposomes for the controlled delivery of paclitaxel: in vitro drug release, cell cytotoxicity, and uptake studies. International journal of nanomedicine, 2011, 6, 151-166.

57. A.Y. Khosroushahi, et al. Novel water-soluble polyurethane nanomicelles for cancer chemotherapy: physicochemical characterization and cellular activities. Journal of nanobiotechnolog, 2012, 10.

58. L. Brannon-Peppas, Polymers in controlled drug delivery. Medical Plastic and Biomaterials, 1997, 4, 34-45.

59. M.D. Louey and L. Garcia-Contreras, Controlled release products for respiratory delivery. American Pharmaceutical Review, 2004, 7, 82-87.

60. P. Bertoglio, SE. Jacobo, and ME. Daraio, Preparation and characterization of PVA films with magnetic nanoparticles: The effect of particle loading on drug release behavior. Journal of applied polymer science, 2010, 115, 1859-1865.

61. S. Nappini, et al. Controlled drug release under a low frequency magnetic field: effect of the citrate coating on magnetoliposomes stability. Soft Matter, 2011, 7, $1025-1037$. 
62. SD. Kong, et al. Magnetic field activated lipid-polymer hybrid nanoparticles for stimuli-responsive drug release. Acta biomaterialia, 2013, 9, 5447-5452 .

63. T. Kobayashi, Cancer hyperthermia using magnetic nanoparticles. Biotechnology journal, 2011, 6, 1342-1347.

64. CS. Kumar, and F. Mohammad, Magnetic nanomaterials for hyperthermia-based therapy and controlled drug delivery. Advanced drug delivery reviews, 2011, 63, 789-808.

65. SH. Emami, et al. The effect of isopropanol addition on enhancement of transdermal controlled release of ibuprofen from ethylene vinyl acetate copolymer membranes. Journal of Applied Polymer Science, 2011, 122, 3048-3054 .

66. P. Costa, and JMS. Lobo, Modeling and comparison of dissolution profiles. European journal of pharmaceutical sciences, 2001, 13, 123-133 .

67. I. Galaev, and B. Mattiasson, Smart polymers: applications in biotechnology and biomedicine. CRC Press, 2007.

68. C. Song, et al. Implications of increased tumor blood flow and oxygenation caused by mild temperature hyperthermia in tumor treatment. International Journal of Hyperthermia, 2005, 21, 761-767.

69. JL. Roti Roti, Cellular responses to hyperthermia (40-46 C): Cell killing and molecular events. International Journal of Hyperthermia, 2008, 24, 3-15.

Caption to Figures:

Fig. 1. The chemical composition of prepared nanoparticles

Fig. 2. XRD pattern of synthesized super paramagnetic iron oxide nanoparticles.

Fig. 3. Grafting palmitoyl groups to the chitosan chain a) synthetic pathway of palmitoyl chitosan b) FT-IR spectra of chitosan and palmitoyl chitosan

Fig. 4. Schematic illustrations of two-step approach for the synthesis of nanoparticles through which SPIONs and Paclitaxel entrapped into the free space

Fig. 5. The physical characteristics of synthesis nanoparticles. a) The hydrodynamic diameter of the drug-free nanoparticles (with/without SPION) b) Zeta potential of nanoparticles before and after loading SPION. c) The hydrodynamic diameter of Paclitaxel loaded nanoparticles. d) Magnetic hysteresis for the coated SPION compared with the naked one. e) Amount of iron oxide incorporated inside chitosan nanoparticles.

Fig. 6. SEM micrographs of a) naked SPION and b) Chitosan-coated SPION

Fig. 7. Paclitaxel loaded nanoparticles' characteristics. a) Drug loading efficiency of nanoparticles (with/without SPION) at an initial drug content of $10 \mathrm{wt} \%$. (Mean $\pm \mathrm{SD}, \mathrm{n}=$ 3 experiments). b) Cumulative in vitro release profiles of Paclitaxel from palmitoyl chitosan nanoparticles (without SPION) and c) composite nanoparticles (with SPION) in the absence and presence of an external magnetic field (Mean $\pm S D, n=3$ experiments). $d$ ) Paclitaxel diffusion coefficients from synthesized nanoparticles.

Fig. 8. Toxicity evaluation of nanoparticles. Drug- free nanoparticles and free paclitaxel in comparison with paclitaxel loaded nanoparticles in the absence and presence of an external magnetic field (Mean $\pm \mathrm{SD}, \mathrm{n}=3$ experiments). 

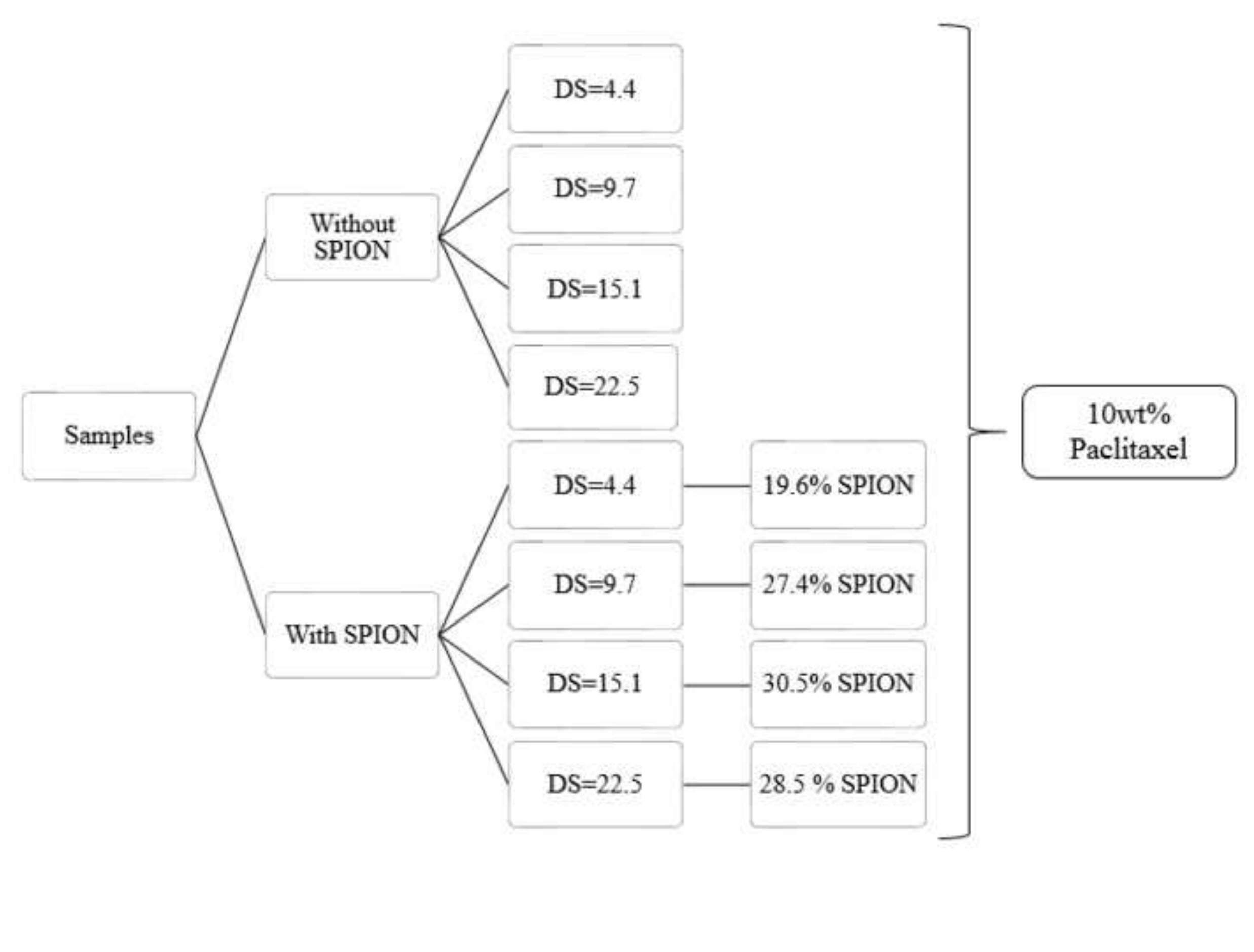


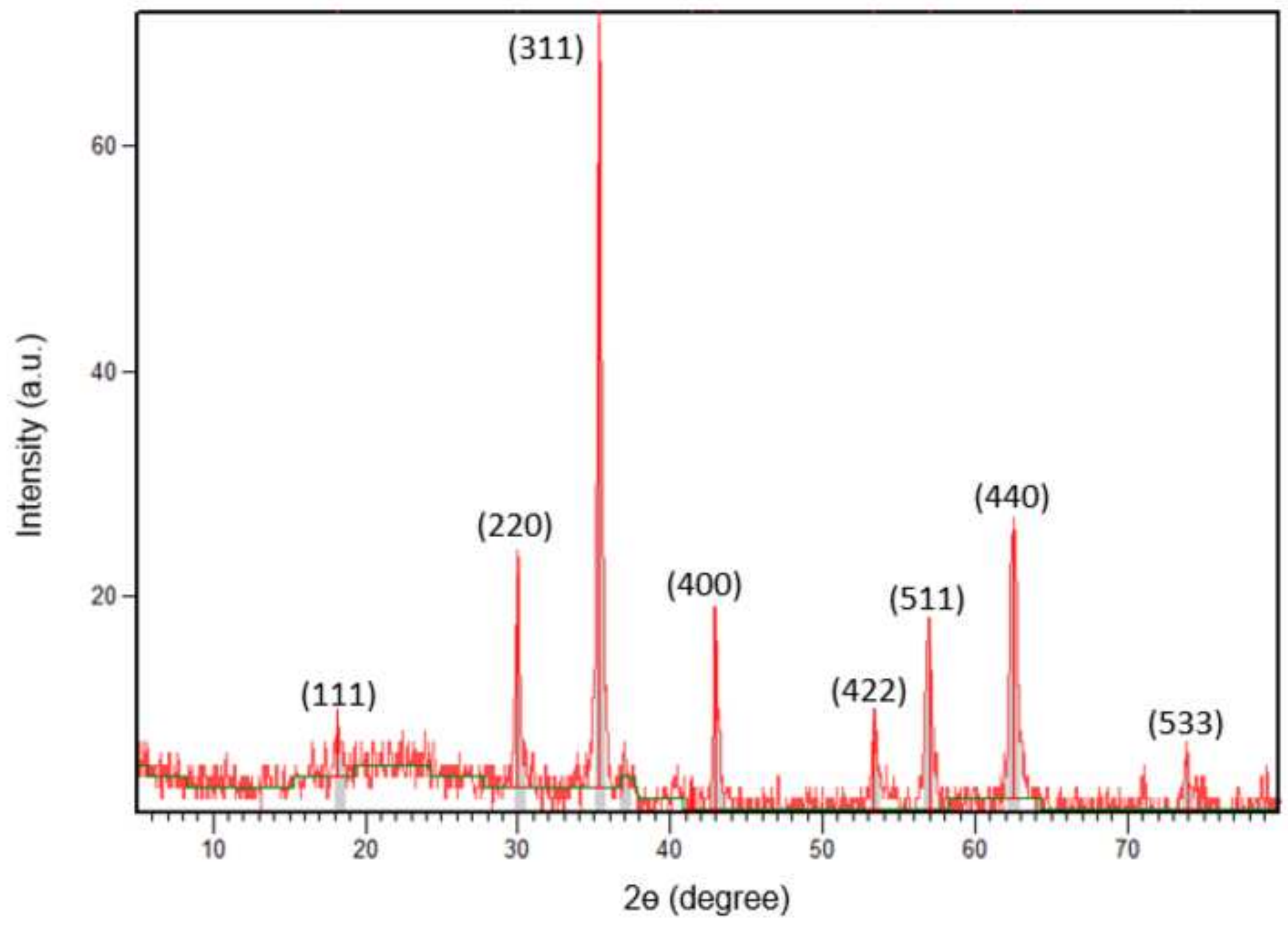



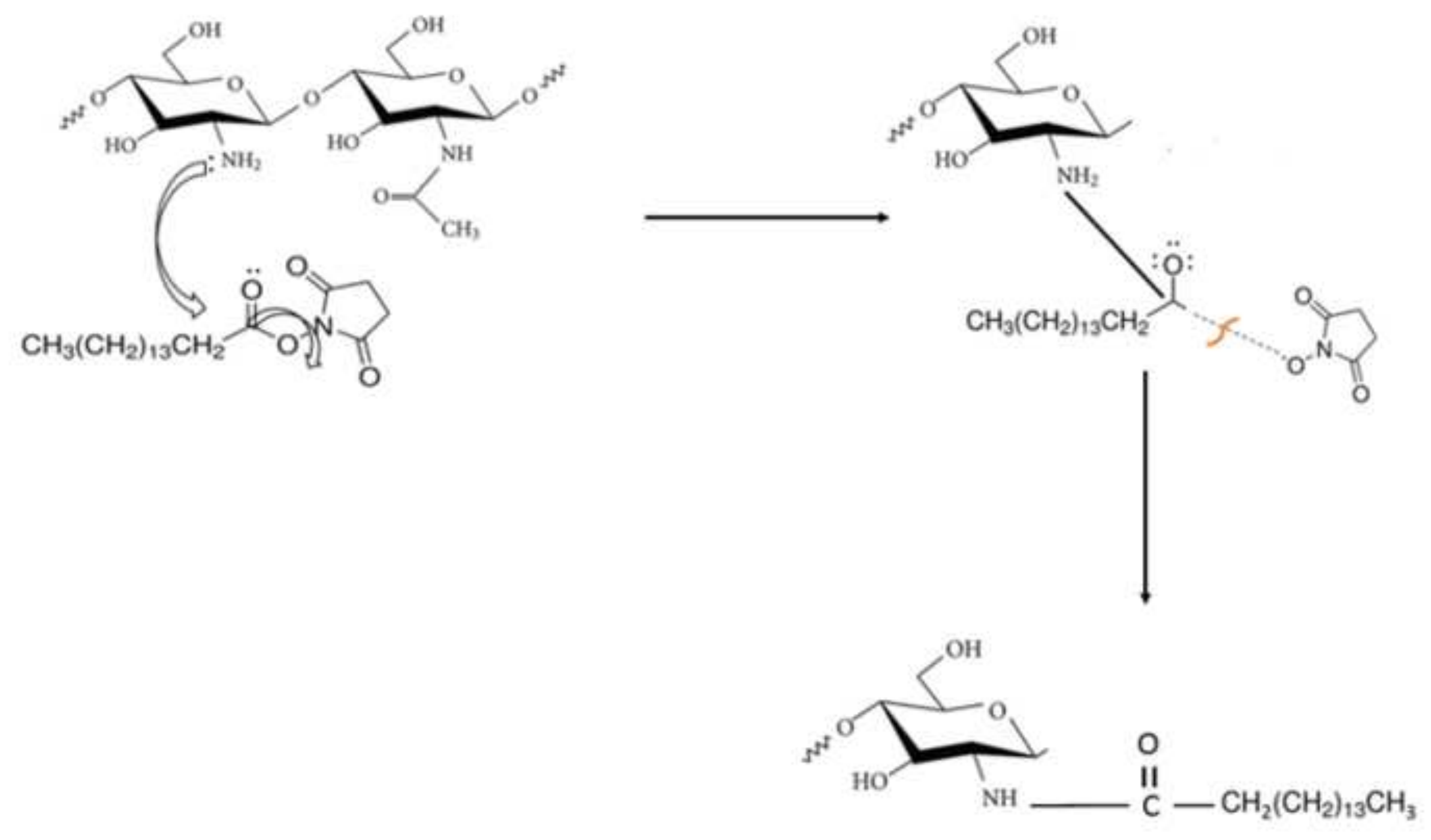


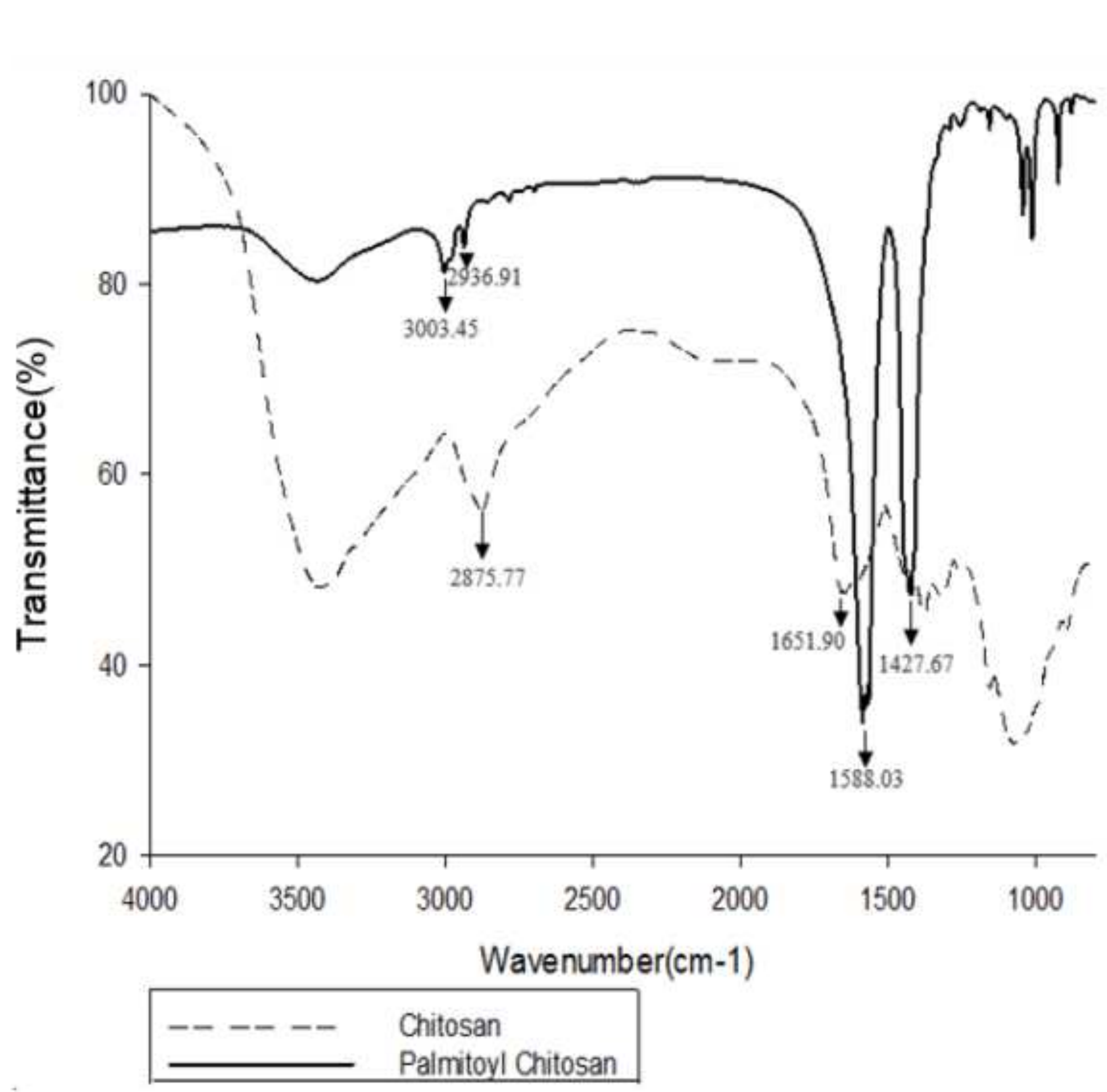



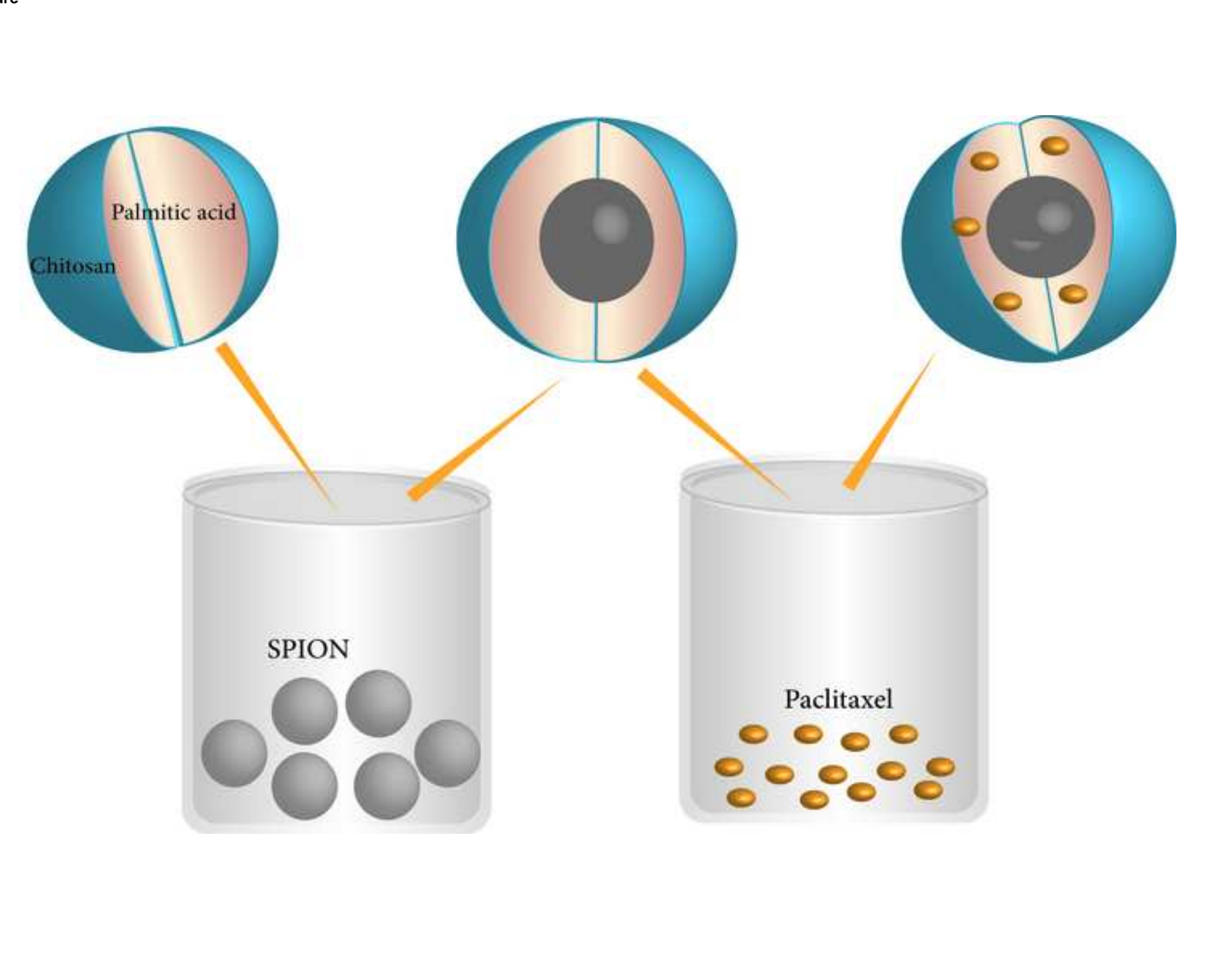

$$
\because 0000
$$
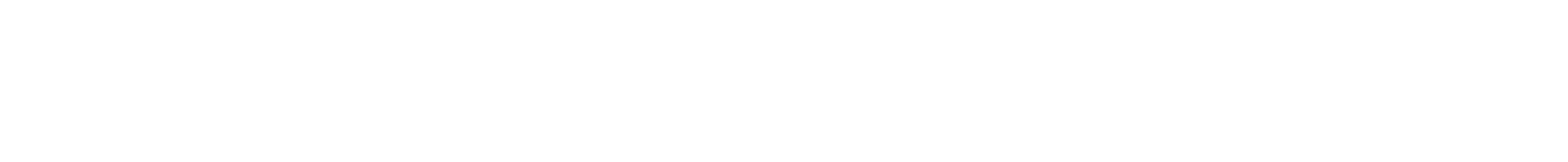


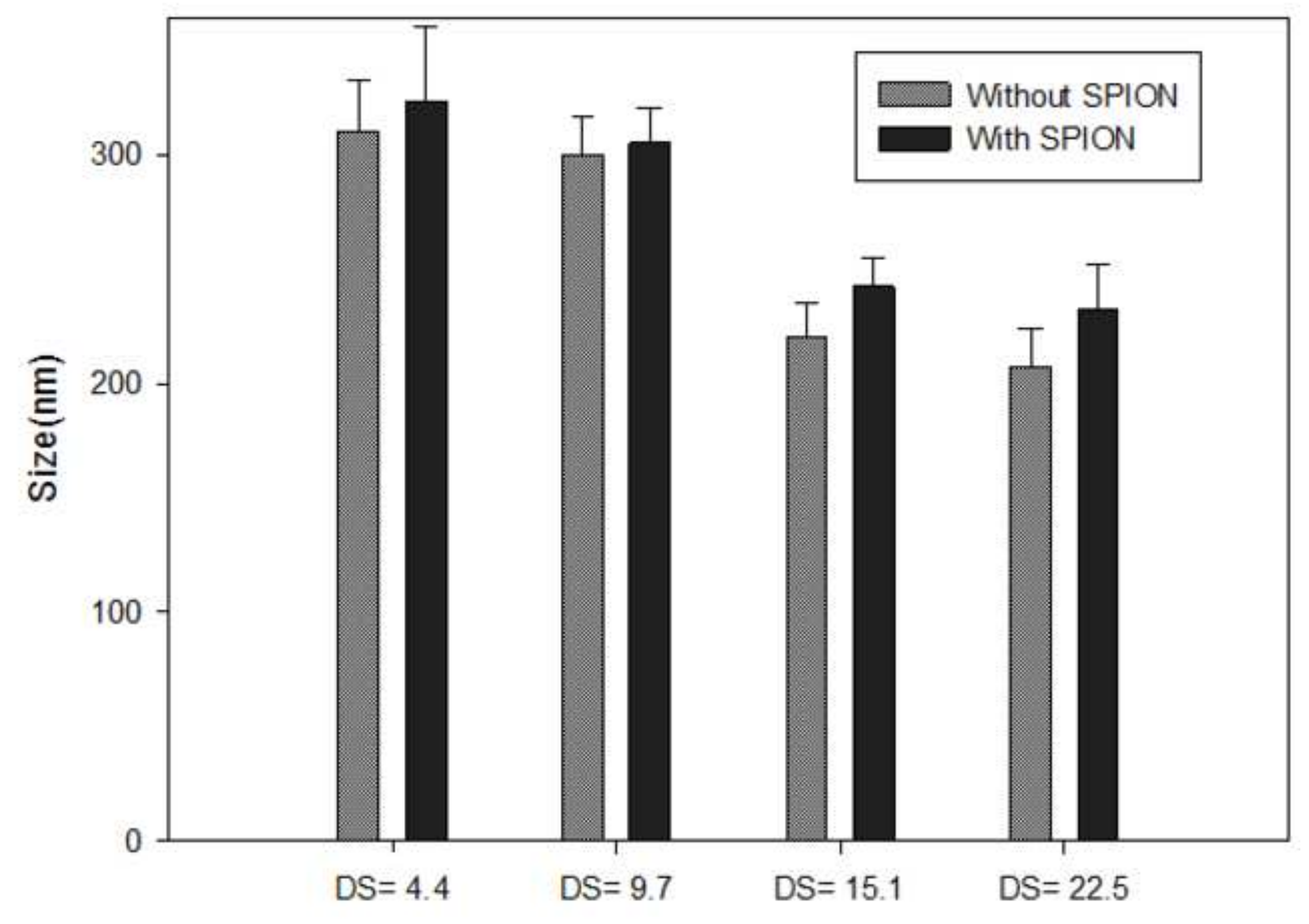

Samples 


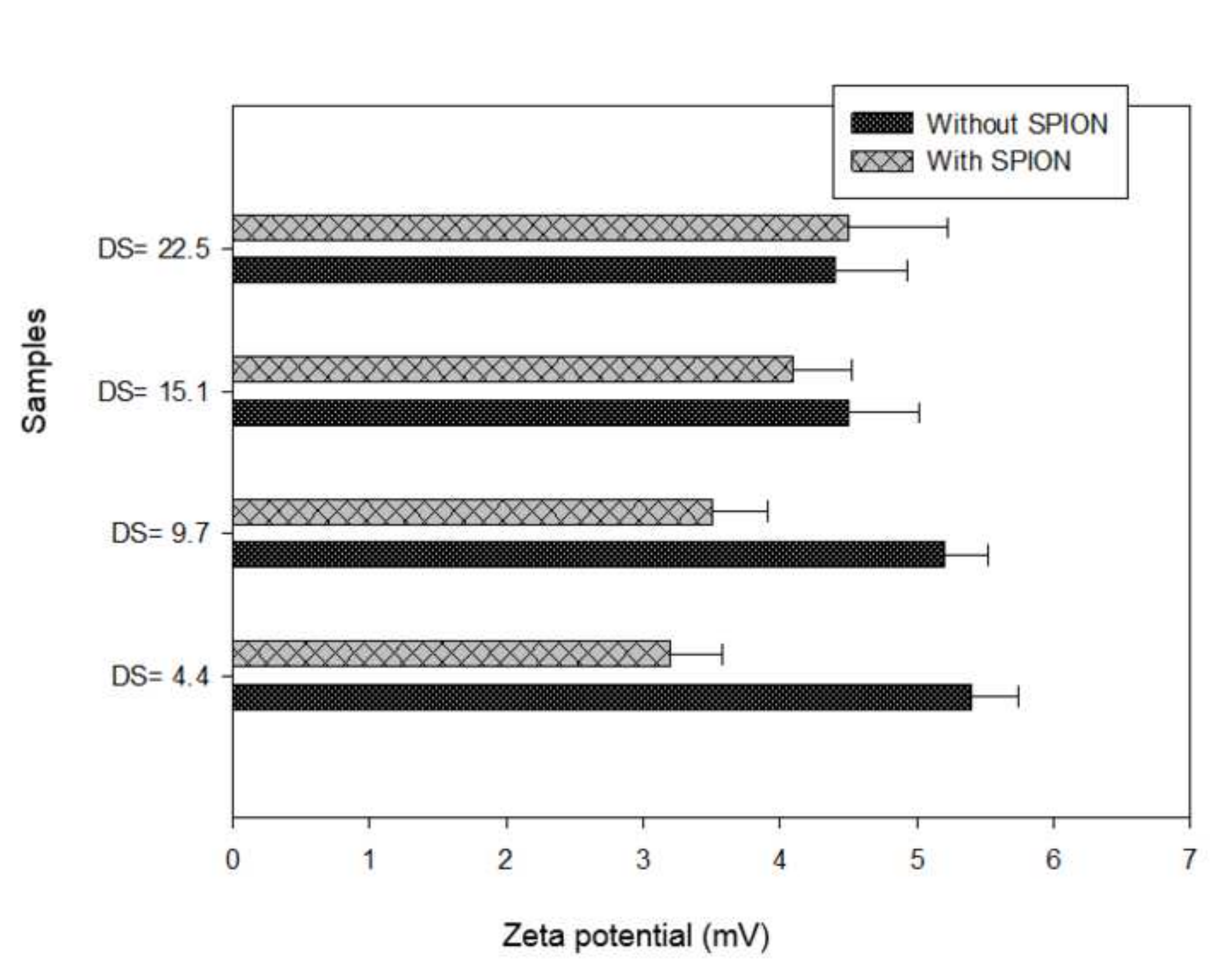

\section{Zeta potential (mV)}

柆

(1)

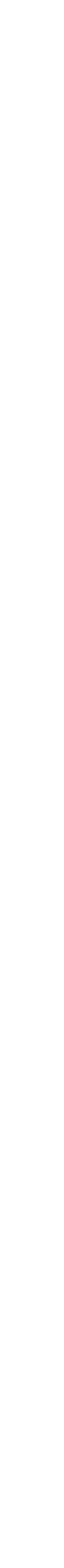




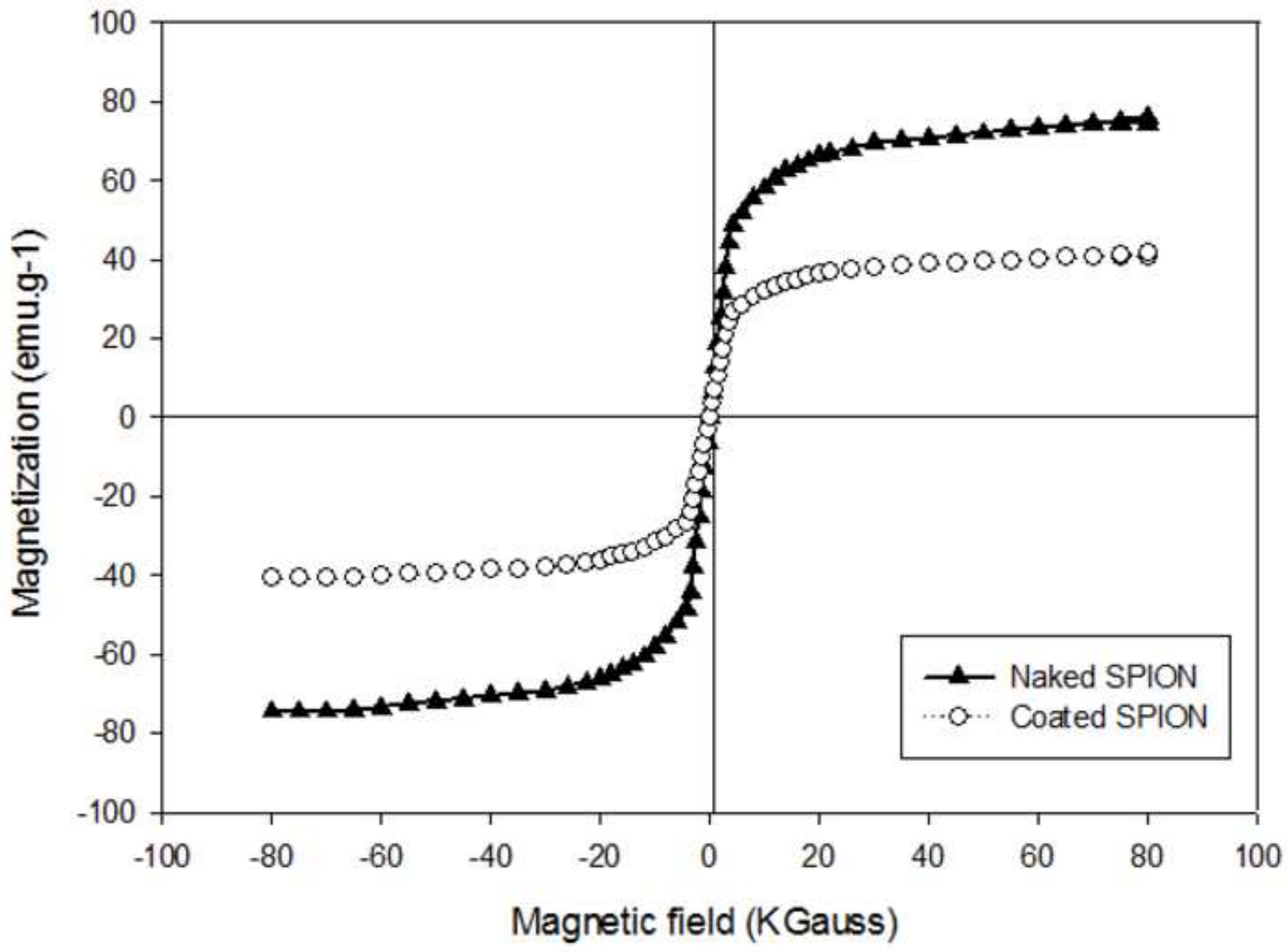




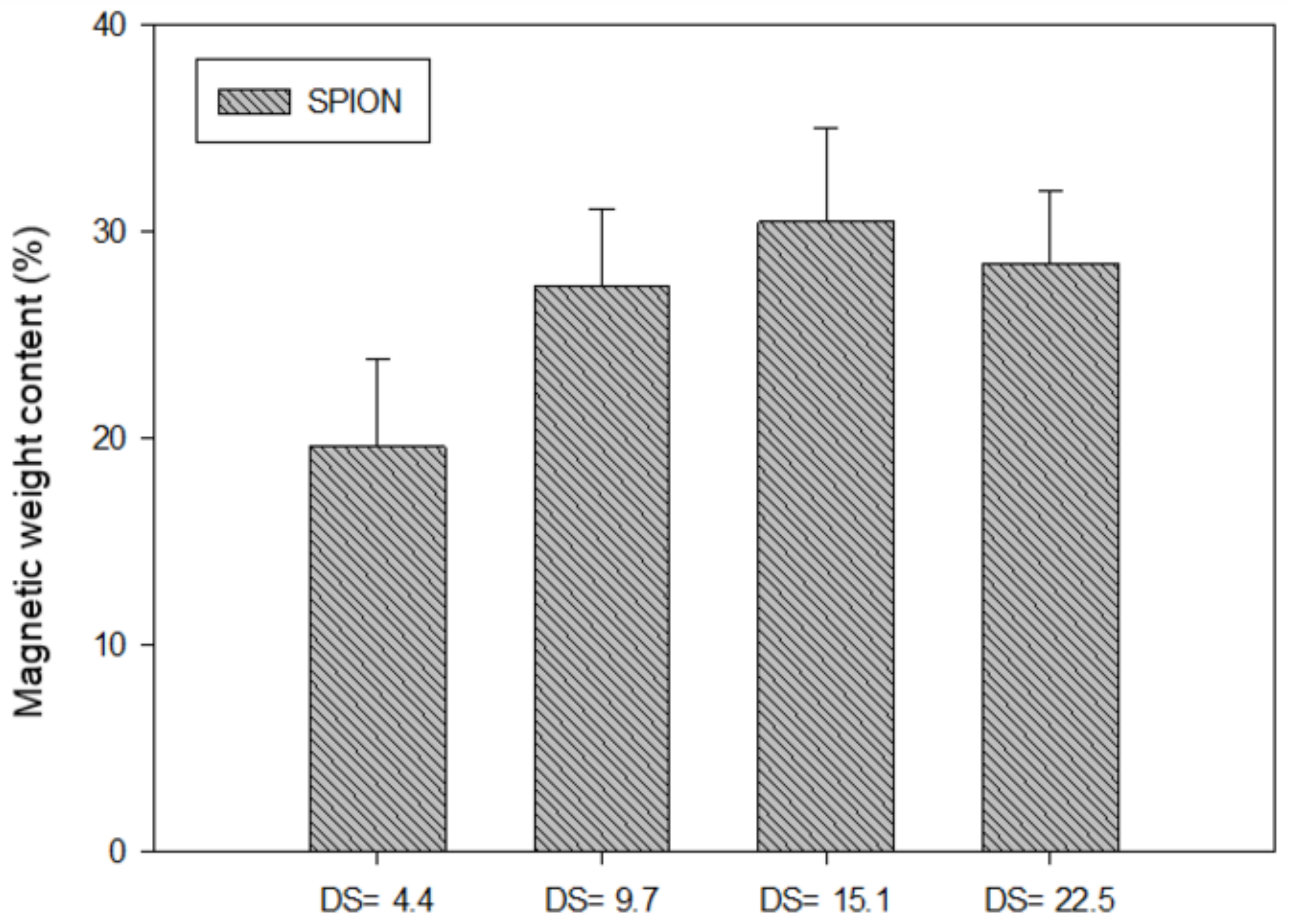

Samples 


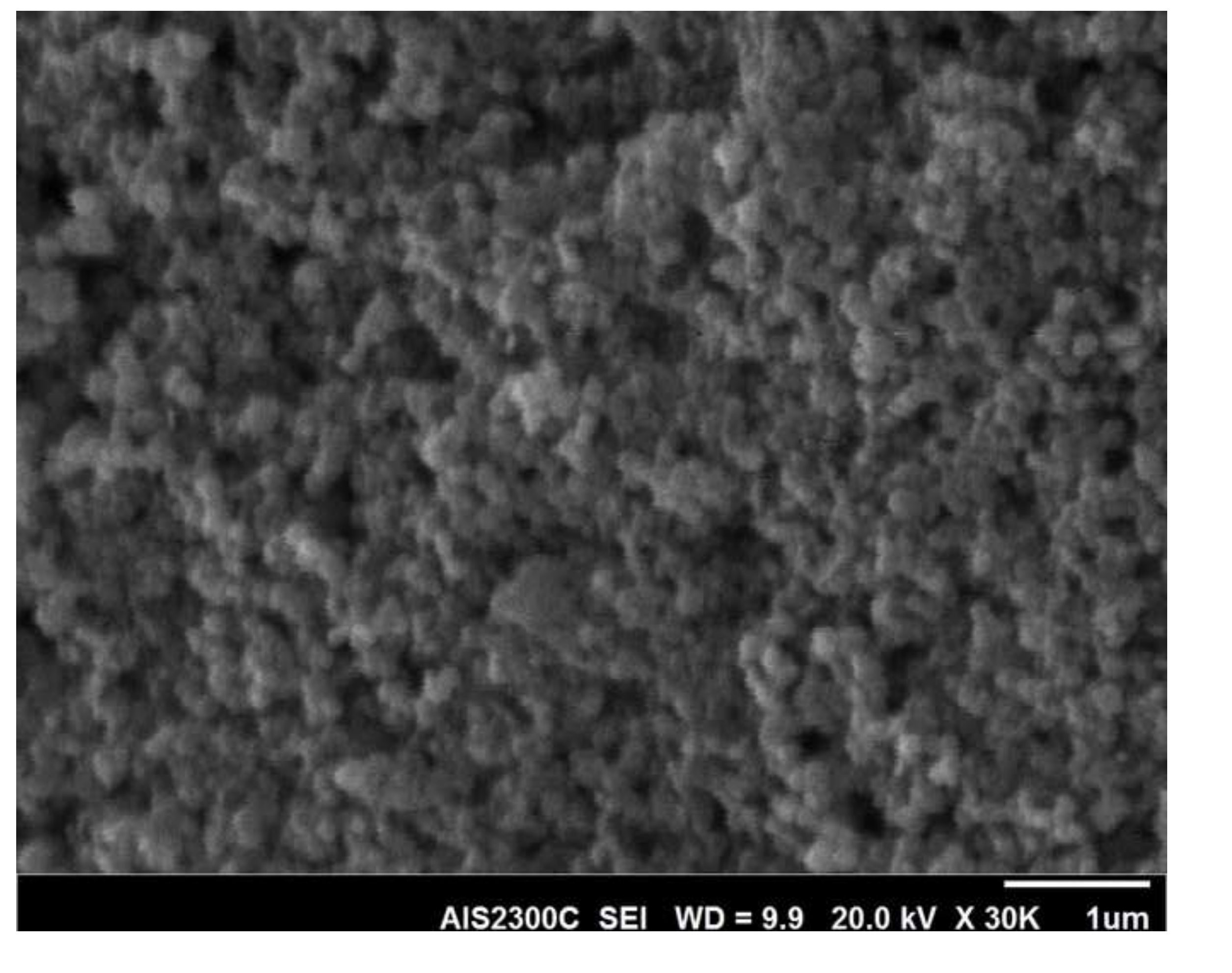




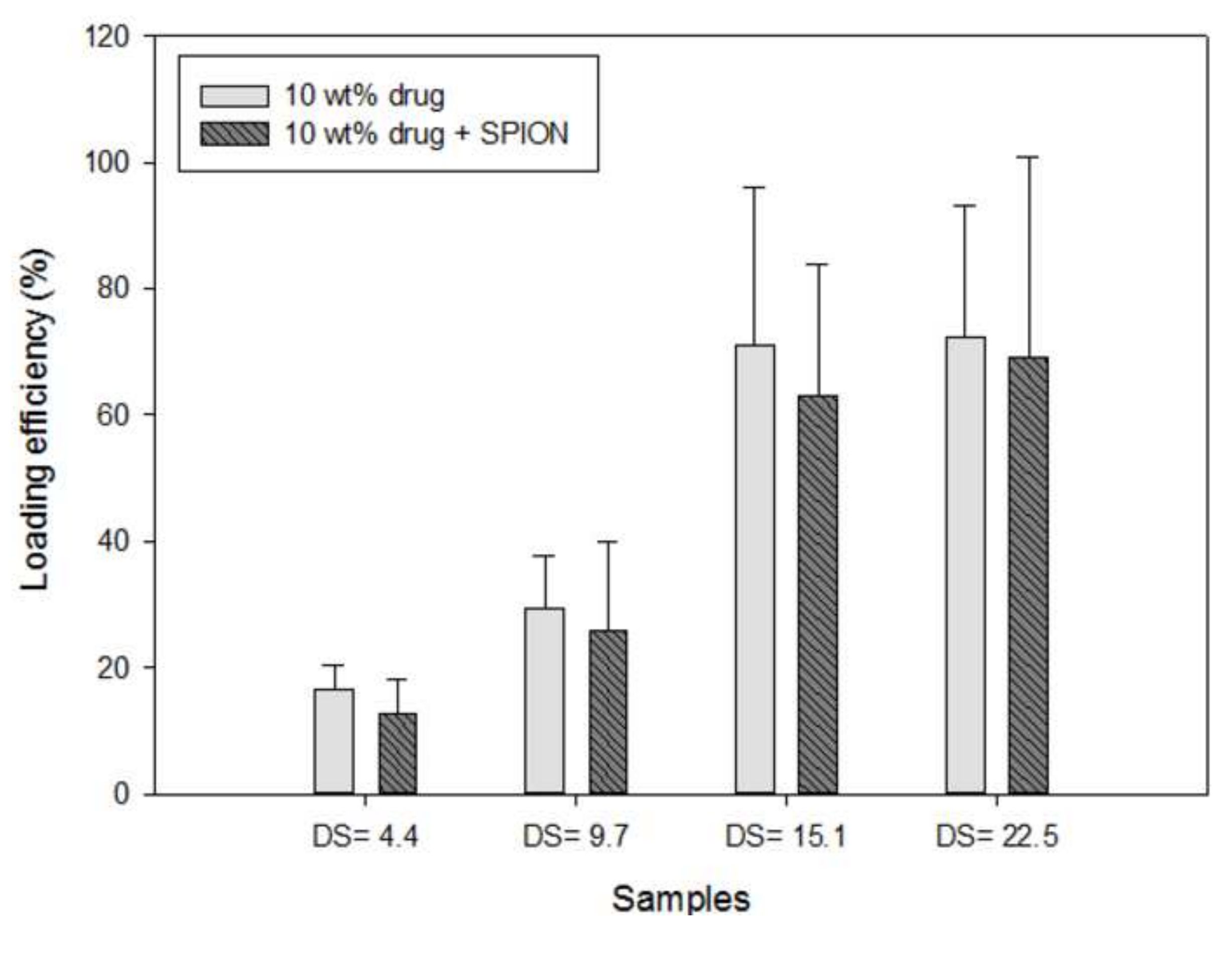

Samples

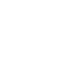
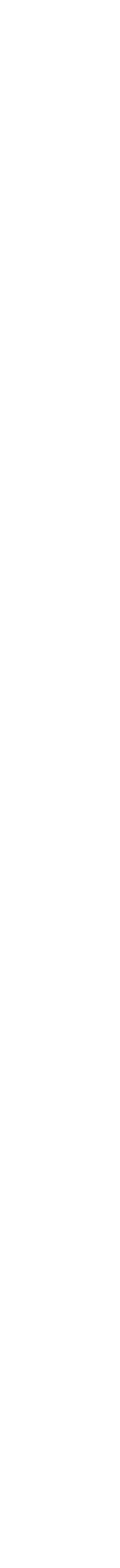

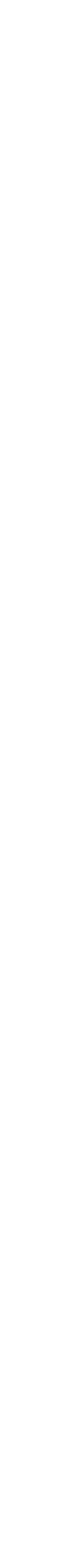




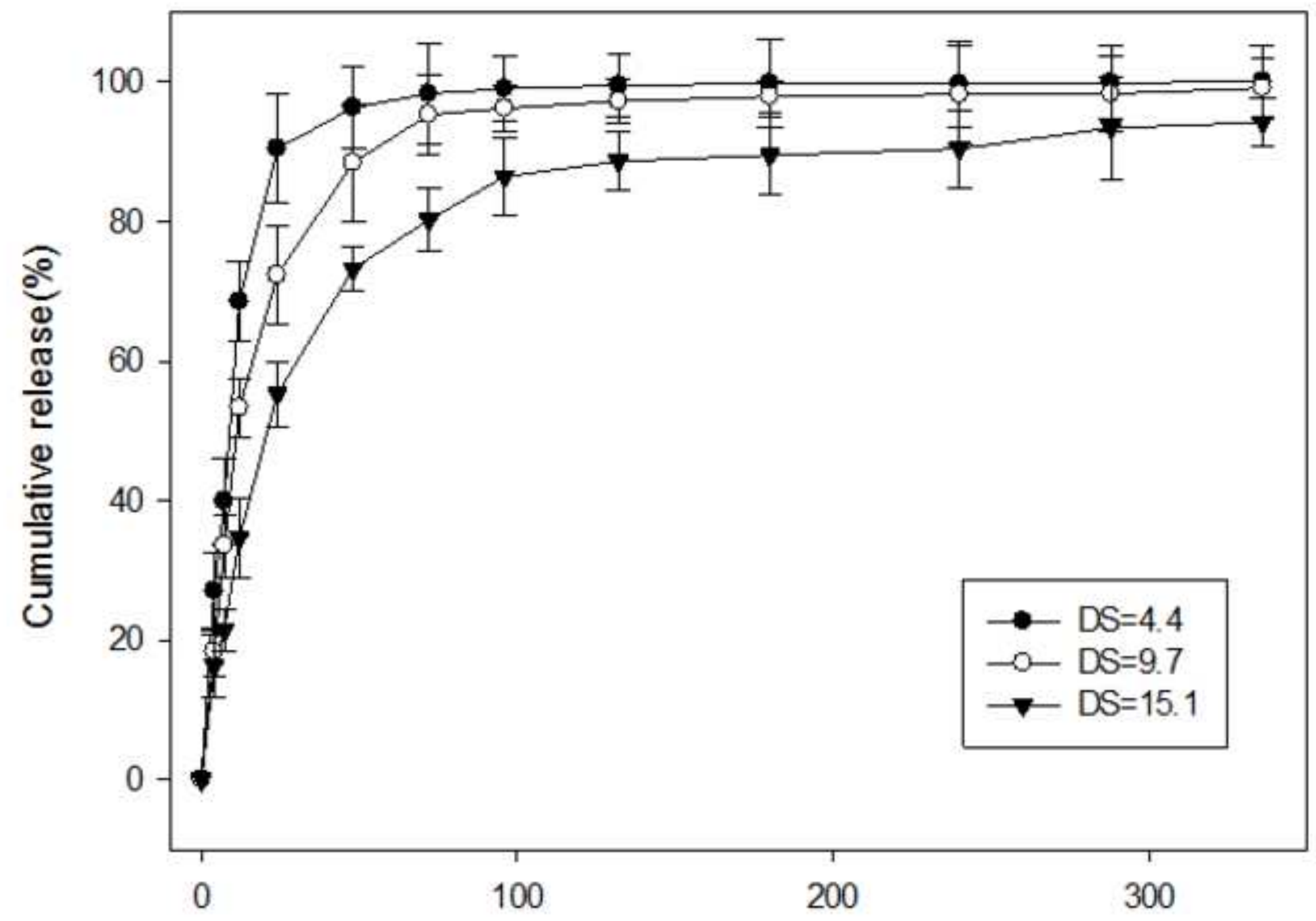

Time (h) 


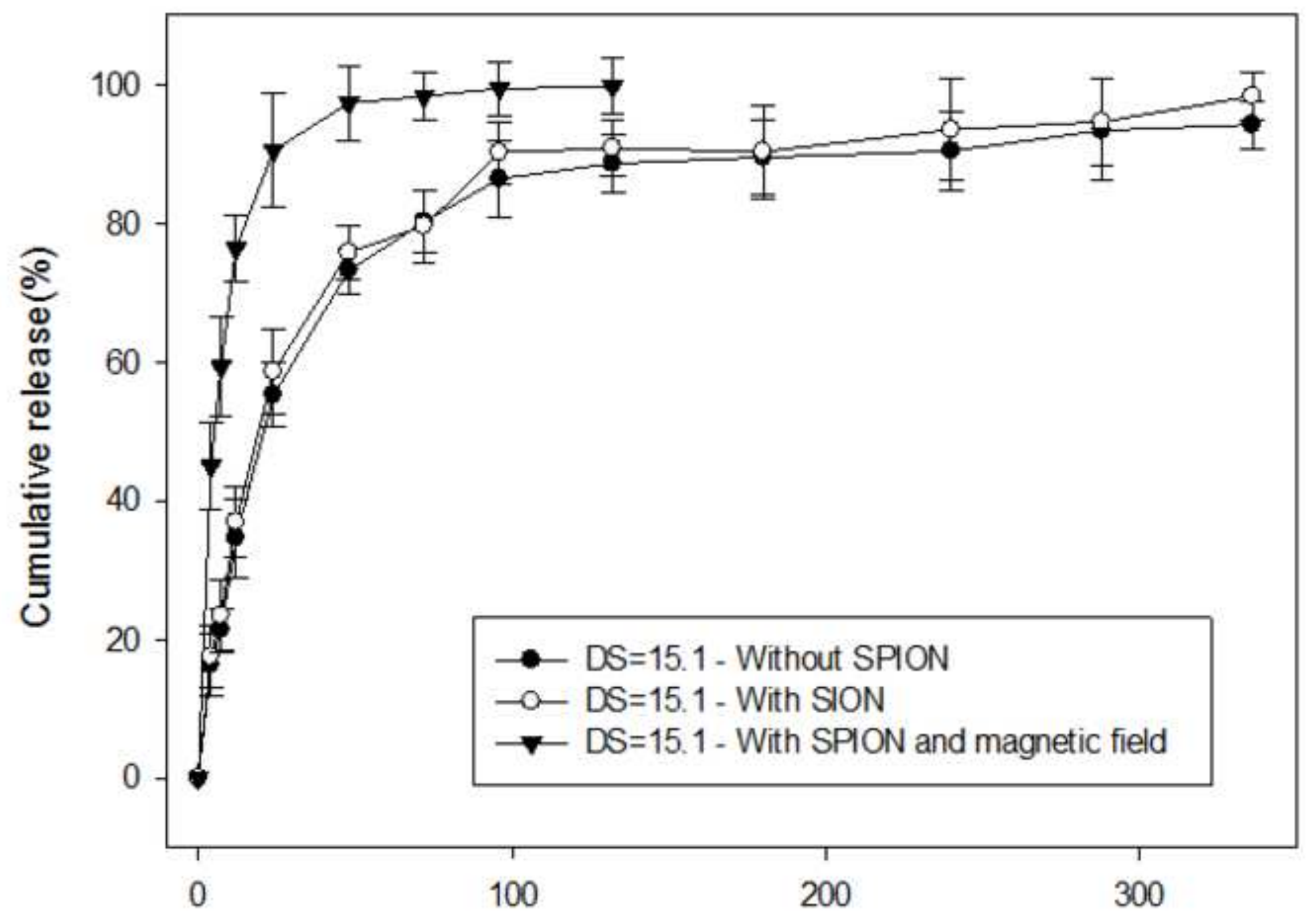

Time (h) 


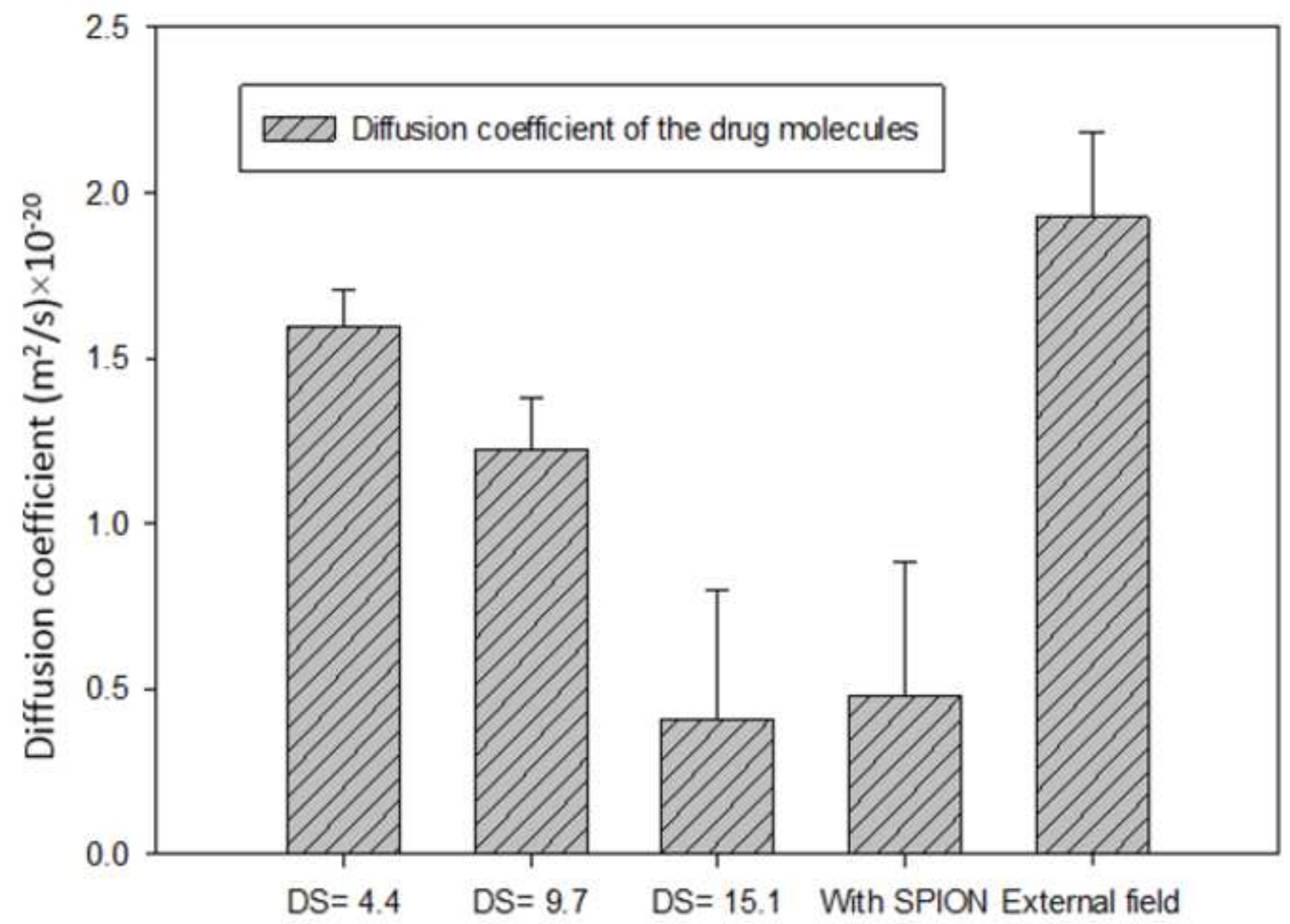




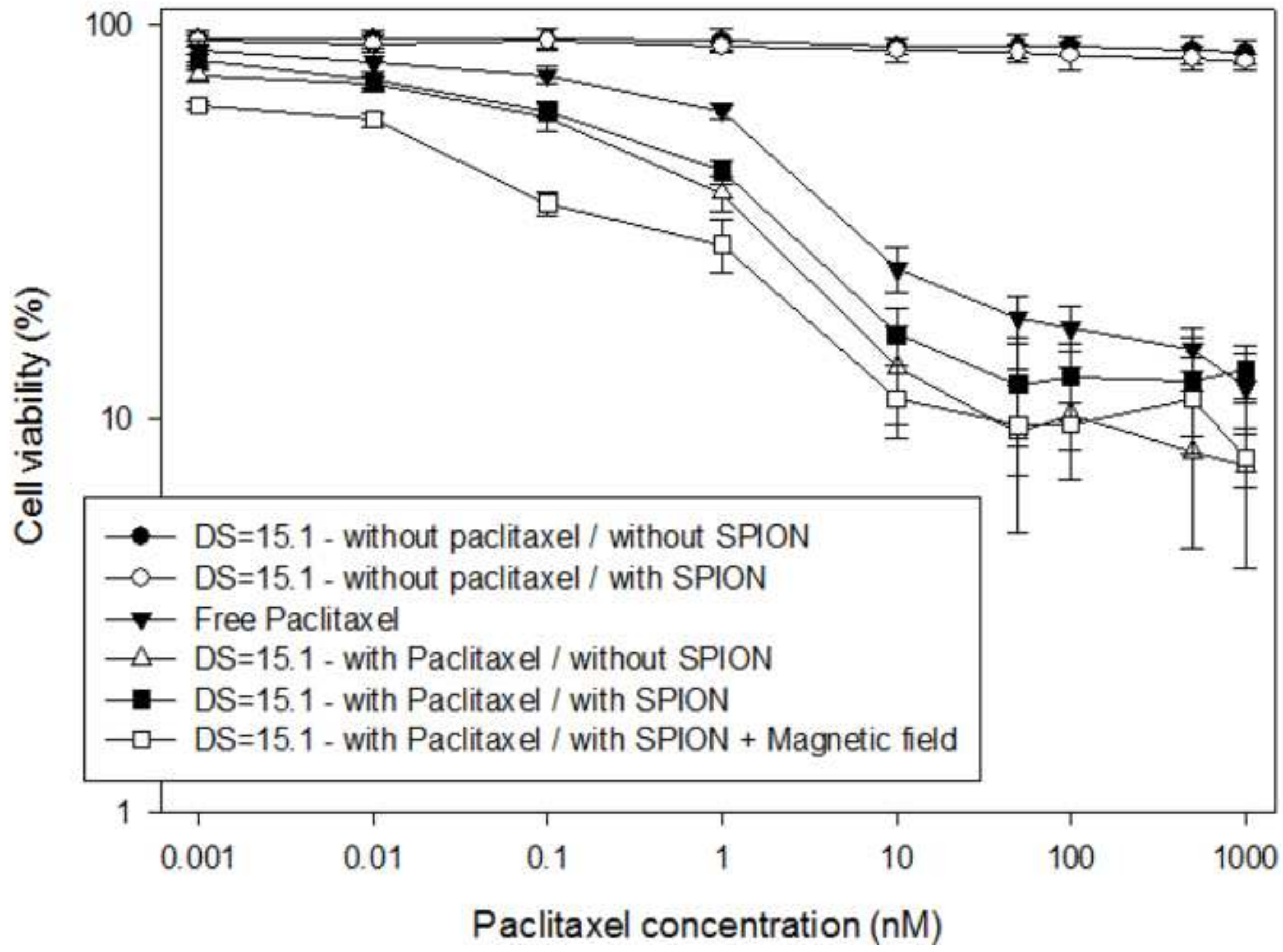




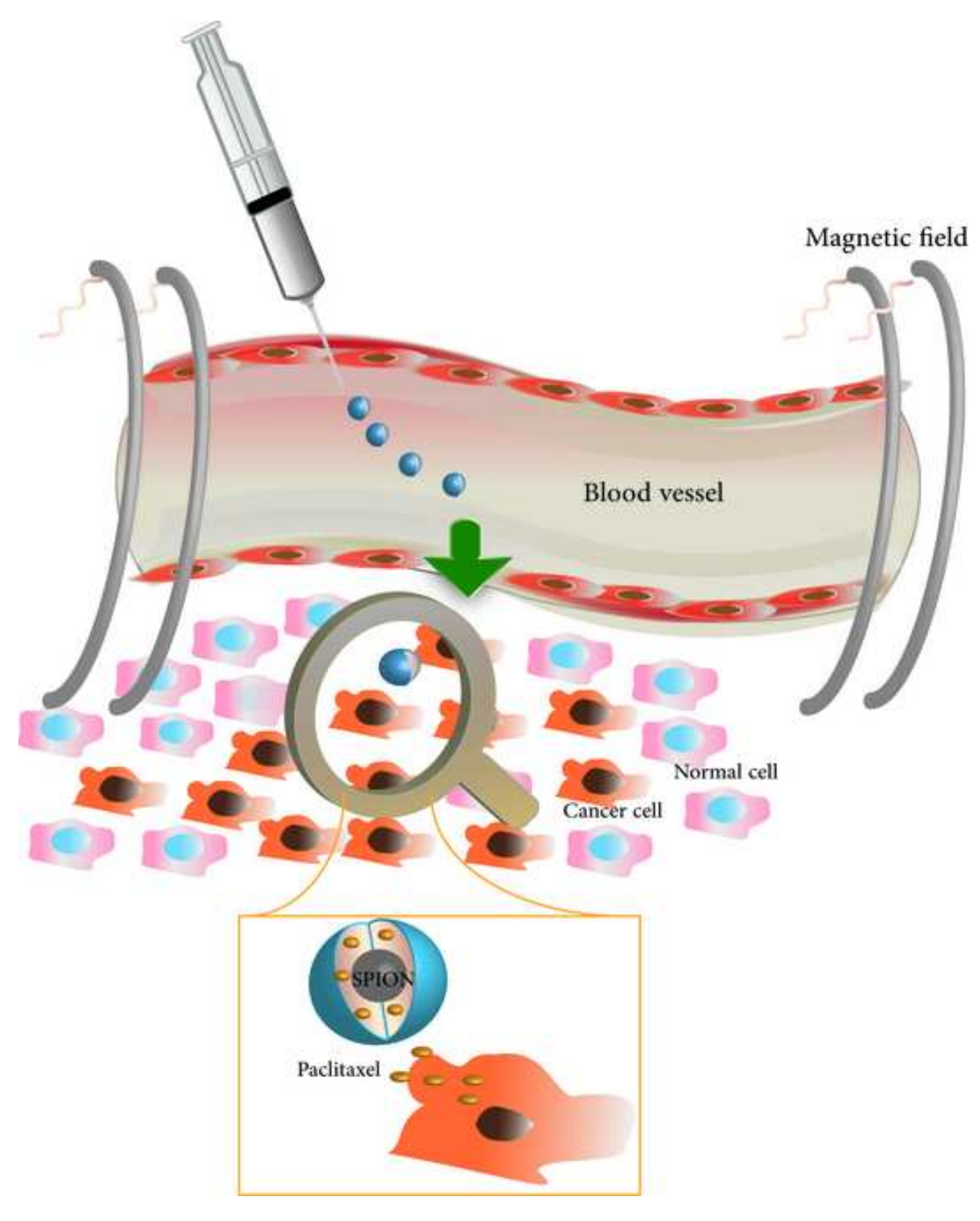

\section{Graphical Abstract}

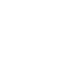

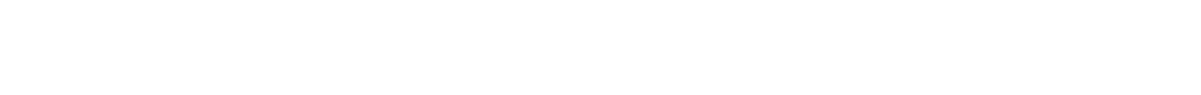

\section{Magnetic field}

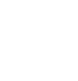

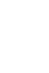

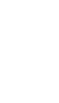

.

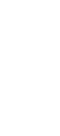

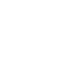

.

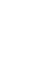

